\title{
On the distortion of turbulence by a progressive surface wave
}

Article

Accepted Version

Teixeira, M. A. C. and Belcher, S. E. (2002) On the distortion of turbulence by a progressive surface wave. Journal of Fluid Mechanics, 458. pp. 229-267. ISSN 0022-1120 doi: https://doi.org/10.1017/S0022112002007838 Available at https://centaur.reading.ac.uk/29255/

It is advisable to refer to the publisher's version if you intend to cite from the work. See Guidance on citing.

Published version at: http://dx.doi.org/10.1017/S0022112002007838

To link to this article DOI: http://dx.doi.org/10.1017/S0022112002007838

Publisher: Cambridge University Press

All outputs in CentAUR are protected by Intellectual Property Rights law, including copyright law. Copyright and IPR is retained by the creators or other copyright holders. Terms and conditions for use of this material are defined in the End User Agreement.

\section{www.reading.ac.uk/centaur}

\section{CentAUR}

Central Archive at the University of Reading

Reading's research outputs online 


\title{
On the distortion of turbulence by a progressive
}

\section{surface wave}

\author{
By M. A. C. TEIXEIRA $\dagger$ \\ AND S. E. B ELCHER \\ Department of Meteorology, University of Reading, \\ Earley Gate, PO Box 243, Reading RG6 6BB, UK
}

(Received ?? and in revised form ??)

A rapid-distortion model is developed to investigate the interaction of weak turbulence with a monochromatic irrotational surface water wave. The model is applicable when the orbital velocity of the wave is larger than the turbulence intensity, and when the slope of the wave is sufficiently high that the straining of the turbulence by the wave dominates over the straining of the turbulence by itself. The turbulence suffers two distortions. Firstly, vorticity in the turbulence is modulated by the wave orbital motions, which leads to the streamwise Reynolds stress attaining maxima at the wave crests and minima at the wave troughs; the Reynolds stress normal to the free surface develops minima at the wave crests and maxima at the troughs. Secondly, over several wave cycles the Stokes drift associated with the wave tilts vertical vorticity into the horizontal direction, subsequently stretching it into elongated streamwise vortices, which come to dominate the flow. These results are shown to be strikingly different from turbulence distorted by a mean shear flow, when 'streaky structures' of high and low streamwise velocity fluctuations develop. It is shown that, in the case of distortion by a mean shear flow, the tendency for the mean shear to produce streamwise vortices by distortion of the turbulent vorticity is largely cancelled by a distortion of the mean vorticity by the turbulent fluctuations. This latter process is absent in distortion by Stokes drift, since there is then no mean vorticity.

The components of the Reynolds stress and the integral length scales computed from turbulence

$\dagger$ Present address: Centro de Geofísica da Universidade de Lisboa, Rua da Escola Politécnica, 58, 1269-102 Lisbon, Portugal. 
distorted by Stokes drift show the same behaviour as in the simulations of Langmuir turbulence reported by McWilliams, Sullivan \& Moeng (1997). Hence we suggest that turbulent vorticity in the upper ocean, such as produced by breaking waves, may help to provide the initial seeds for Langmuir circulations, thereby complementing the shear-flow instability mechanism developed by Craik \& Leibovich (1976).

The tilting of the vertical vorticity into the horizontal by the Stokes drift tends also to produce a shear stress that does work against the mean straining associated with the wave orbital motions. The turbulent kinetic energy then increases at the expense of energy in the wave. Hence the wave decays. An expression for the wave attenuation rate is obtained by scaling the equation for the wave energy, and is found to be broadly consistent with available laboratory data.

\section{Introduction}

The uppermost layer of the ocean has three interacting dynamical components, namely a winddriven mean shear current, surface waves and turbulence. This potent mixture produces a variety of intriguing phenomena that do not occur in the atmospheric boundary layer. Observations in the ocean mixed layer by Faller \& Auer (1988) have revealed elongated streamwise vortices, identified as Langmuir circulations, with a wide range of scales, which can therefore be seen as a type of turbulence, named Langmuir turbulence by McWilliams, Sullivan \& Moeng (1997). Furthermore, recent observations show that breaking waves are a surprisingly potent source of turbulence in the upper few metres of the mixed layer (Agrawal et al. 1992; Terray et al. 1996). Given the complexity of this system it is helpful to consider interactions between pairs of these components. Such idealised calculations are presented in this paper.

Turbulence and surface waves can interact in a variety of ways. Turbulent pressure fluctuations and turbulent shear stresses are responsible for both the initiation of surface waves (Phillips 1957; Teixeira 2000) and their subsequent amplification by a sheltering mechanism (Belcher \& Hunt 1993, 1998). Turbulence in the water can scatter surface waves (Phillips 1959), distort surface waves (Longuet-Higgins 1996) and dissipate surface waves (Kitaigorodskii \& Lumley 1983). Conversely, 
breaking waves, often visible as whitecaps (Melville 1996), shear currents induced by the wind, and thermal convection all generate turbulence in the ocean surface layer, which is subsequently distorted by orbital motions associated with the surface waves (Thais \& Magnaudet 1996). More idealised interactions between waves and turbulence have been investigated experimentally by Green, Medwin \& Paquin (1972), Ölmez \& Milgram (1992), Nepf et al. (1995) and Thais \& Magnaudet (1996). But a systematic theoretical treatment of this situation is lacking.

Distortion of turbulence by surface waves is of two types: on the one hand, there is the direct effect of the orbital motions, of first order in the wave slope, which has a straining rate of $O\left(a_{w} k_{w} \sigma_{w}\right)$, where $a_{w}, k_{w}$ and $\sigma_{w}$ are, respectively, the amplitude, wavenumber and angular frequency of the waves. This effect is relatively weak, because the wave motions are periodic and the total strain never exceeds $O\left(a_{w} k_{w}\right)$, which is small. On the other hand, there is the effect of the Stokes drift, of second order in the wave slope, whose straining rate can be estimated as $O\left(a_{w}^{2} k_{w}^{2} \sigma_{w}\right)$. Although this straining rate is even smaller, its effect is cumulative, and the total strain is of $O\left(a_{w}^{2} k_{w}^{2} \sigma_{w} t\right)$, where $t$ is time. So, this second order effect is bound to affect turbulence appreciably after a sufficient number of wave cycles.

Interaction between the wind-driven mean shear current and the Stokes drift is at the heart of current explanations of the generation of Langmuir circulations in the ocean (Leibovich 1983). Langmuir circulations are intense, elongated vortices, with their axes of rotation roughly aligned with the wind and the dominant surface waves. Craik \& Leibovich (1976) propose two mechanisms for the production of Langmuir circulations based on instability of the mean shear current under the action of the wave motions. The first, known as CL1 or the direct drive mechanism, involves interaction between the mean shear current in the water and the Stokes drift associated with waves propagating at an angle to this mean current. The second mechanism for production of Langmuir circulations analysed by Craik \& Leibovich (1976), known as the CL2 mechanism, involves tilting and stretching of vertical vorticity into the horizontal by the Stokes drift associated with surface waves propagating in the same direction as the mean shear current. In the Craik-Leibovich formulation the vertical vorticity is assumed to arise from infinitesimal spanwise variations in the mean shear current. 
The mechanisms for generation of Langmuir circulations developed by Craik \& Leibovich (1976) treat explicitly interactions between the mean shear flow and the Stokes drift. The role of turbulence is represented by an eddy viscosity, thereby relegating turbulence to a mechanism for dissipating the Langmuir circulations. But there is evidence that turbulence may contribute to the generation of Langmuir circulations (Nepf et al. 1995). Hence one aim of the present study is to quantify the effects of Stokes drift on turbulence in the water flow.

We focus here on two idealised model problems that aim at clarifying the interactions in the upper ocean. Firstly, the bulk of the paper is concerned with the distortion of turbulence by the passage of a surface wave; the mean shear flow is neglected. This distortion is traced from the periodic straining of the wave, through to the cumulative distortion of the turbulence by the Stokes drift, to decay of waves as energy is transferred to the turbulence. Secondly, we present results of distortion of turbulence by a mean shear flow; the effects of the surface waves are neglected. Comparison of the results of these two analyses graphically shows the important role played by Stokes drift in shaping the turbulence and offers clues to an additional source of Langmuir circulations.

The remainder of this paper is organised as follows: in $\S 2$, the theoretical model of waveturbulence interaction is presented. Section 3 presents the resulting time evolution of the Reynolds stresses and the integral length scales of the turbulence, firstly over a single wave cycle and secondly over several wave cycles, when the Stokes drift becomes important. These latter results are compared with turbulence distortion by a mean shear. The overall budgets of kinetic energy in the turbulence and the wave motions are then analysed to show how the waves decay. The paper ends with the main conclusions, in $\S 4$.

\section{Theoretical model}

Consider a semi-infinite water mass bounded above by a free surface on which a progressive, monochromatic, surface wave is propagating. The wave is irrotational and has relatively low slope. Here we analyse how turbulence beneath the free surface is distorted by the orbital motions associated with the wave. To tackle this idealised problem, the rapid-distortion theory (RDT) of Batchelor \& Proudman (1954) and Hunt (1973) will be used. 
The total velocity field $\mathcal{U}_{i}$ is split into a mean part, $U_{i}$, and a turbulent part, $u_{i}$, namely

$$
\mathcal{U}_{i}=U_{i}(\boldsymbol{x})+u_{i}(\boldsymbol{x}, t), \quad i=1,2,3 .
$$

The mean velocity corresponds to the wave orbital motion expressed in a coordinate system travelling with the wave crests: hence $U_{i}$ is stationary. The wave is taken to propagate in the positive $x_{1}$ direction and, since the wave is also monochromatic, $U_{i}$ is slab-symmetric and does not depend on $x_{2}$. The turbulence is assumed to be statistically stationary, homogeneous and isotropic far from the surface. At the initial time, the turbulence is assumed to be homogeneous and isotropic throughout the depth of the water column at a particular point of the wave phase. The turbulence is also assumed to be of a much smaller scale than the wave, so that the initial integral length scale of the turbulence $l$ satisfies $l \ll \lambda_{w}$, where $\lambda_{w}$ is the wavelength of the wave. The idea is then to analyse the evolution of the turbulence statistics following a water parcel as the wave propagates over the turbulence.

This idealised model approximates two physical situations: the first is a laboratory arrangement where mechanically generated surface waves propagate over a region of turbulence created by an oscillating grid, as in the experiments of Ölmez and Milgram (1992) and Milgram (1998). The second situation is where turbulence is injected into the water by breaking surface waves at a particular time and location, being then distorted by subsequent waves. Of course, in the second case, the initial turbulence is not perfectly homogeneous and isotropic, but it is reasonably isotropic (Rapp \& Melville 1990) and its integral length scale is generally considerably smaller than the wavelength of the dominant waves, since wave breaking is a highly localised process. Hence the condition $l \ll \lambda_{w}$ is probably satisfied.

In the formulation adopted by Hunt (1973), RDT is based on the inviscid equations of motion, linearised with respect to the turbulence. For example, the linearised vorticity equation can be written

$$
\frac{\partial \omega_{i}}{\partial t}+U_{j} \frac{\partial \omega_{i}}{\partial x_{j}}+u_{j} \frac{\partial \Omega_{i}}{\partial x_{j}}=\Omega_{j} \frac{\partial u_{i}}{\partial x_{j}}+\omega_{j} \frac{\partial U_{i}}{\partial x_{j}},
$$

where $\boldsymbol{\Omega}=\nabla \times \boldsymbol{U}$ is the vorticity of the mean flow and $\omega=\nabla \times \boldsymbol{u}$ is the vorticity of the turbulence. In the present case, $U_{i}$ is the velocity associated with an irrotational surface wave, so $\Omega_{i}=0$. If $u$ is defined as the initial root-mean-square (RMS) velocity of the turbulence, $U$ as the typical 
velocity scale of the mean flow and $L$ as the typical length scale over which the mean flow varies, the conditions for the validity of (2.2) are that the turbulent velocity is sufficiently weak compared with the mean velocity, $u \ll U$, and that the strain rate of the mean flow is higher than that associated with the interaction of the turbulence with itself, $u / l \ll U / L$. The first condition is immediately satisfied if, additionally to the second, $l \ll L$ is also satisfied. It will be seen later that this last condition on the length scales is convenient if the equations of motion are to be simplified by being expressed in a curvilinear coordinate system aligned with the mean flow (Durbin \& Hunt 1980). In that case, the curvature terms in the equations are of $O(l / L)$ and can be neglected, i.e. the equations take at leading order the same form as in a Cartesian coordinate system.

For the particular flow under consideration, the length scale of the mean flow is the wavelength of the wave, $\lambda_{w}$, and the strain rate associated with the wave is of $O\left(a_{w} k_{w} \sigma_{w}\right)$, so the conditions for which the linear RDT model is valid are

$$
l \ll \lambda_{w}, \quad a_{w} k_{w} \gg \frac{u}{l \sigma_{w}} .
$$

Hence, the turbulence has to be of relatively small scale and the steepness of the wave cannot be too small.

Turbulence generated by a grid in laboratory experiments (Brumley and Jirka 1987; Kit, Strang \& Fernando 1997) or associated with a wind-induced shear current (Melville, Shear \& Veron 1998) generally has an integral length scale of $O(1 \mathrm{~cm})$ or larger. Turbulence generated by breaking waves is likely to be even larger (see table V of Kitaigorodskii et al. 1983). Since the transition between the gravity and capillary regimes of surface waves occurs at a wavelength $\approx 1.7 \mathrm{~cm}$, the first condition of (2.3) is typically satisfied if the wave that distorts the turbulence is a gravity wave. Then, if it is noted that, in the deep-water gravity wave regime, the dispersion relation gives $\sigma_{w}=\left(2 \pi g / \lambda_{w}\right)^{\frac{1}{2}}$, the second equation of (2.3) may also be expressed as a condition on the wavelength, and (2.3) takes the more compact form

$$
l \ll \lambda_{w} \ll 2 \pi g\left(\frac{l}{u} a_{w} k_{w}\right)^{2}
$$

Taking the reasonable values $l=5 \mathrm{~cm}, u=1 \mathrm{~cm} \mathrm{~s}^{-1}, a_{w} k_{w}=0.1$, and $g=9.8 \mathrm{~m} \mathrm{~s}^{-1}$, the following 
estimate for the range of applicability of the model is obtained:

$$
5 \mathrm{~cm} \ll \lambda_{w} \ll 15.4 \mathrm{~m} .
$$

This condition is easily satisfied for laboratory waves, and for an important fraction of the gravity waves existing in the ocean. The scalings of Belcher et al. (1994) confirm that the RDT assumption is valid in the water flow in most situations, except very near the interface.

\subsection{Mathematical formulation}

In applying RDT to mean flows as complex as a progressive surface wave, it is convenient to express the vorticity equation (2.2) in the intrinsically Lagrangian form due to Cauchy (Batchelor \& Proudman 1954),

$$
\omega_{i}(\boldsymbol{x}, t)=\frac{\partial x_{i}}{\partial a_{j}} \omega_{j}(\boldsymbol{a}, 0)
$$

where

$$
x_{i}(\boldsymbol{a}, t)=a_{i}+\int_{0}^{t} U_{i}\left(\boldsymbol{x}, t^{\prime}\right) \mathrm{d} t^{\prime}
$$

is the position at time $t$ of a fluid parcel with initial position $a_{i} . \partial x_{i} / \partial a_{j}$ is the strain tensor, which gives the ratio of the separations, along the 3 coordinate directions, of two infinitesimally distant material particles at a given time, following the fluid motion, and at the initial time. In (2.6), the Cauchy equation is already linearised, like (2.2), because that is the form relevant for the present RDT problem, and $\Omega_{i}=0$ has been assumed, because the wave is irrotational. If the mean flow was not irrotational, (2.6) would have to include $\Omega_{i}$ as well, and the strain tensor would include the distortion of the mean vorticity by the turbulent velocity, as pointed out recently by Nazarenko, Kevlahan \& Dubrulle (1999). This would make the calculations much more complicated.

Given the initial turbulent velocity field $u_{i}(\boldsymbol{a}, 0)$, the initial turbulent vorticity $\omega=\nabla \times \boldsymbol{u}$ is obtained by taking the curl and, once the strain tensor is known, the final velocity field may be recovered from the final vorticity obtained from (2.6) by solving the equation

$$
\nabla^{2} \boldsymbol{u}=-\nabla \times \omega
$$

which results from taking the curl of the definition of turbulent vorticity. The remaining problem, therefore, is determining $\partial x_{i} / \partial a_{j}$ as a function of the mean velocity field.

Durbin (1978) noted that the form taken by the strain tensor is considerably simplified if the 
RDT problem is formulated in a streamline coordinate system. In fact, such formulation is not only advantageous for simplifying the form taken by the equations of motion but also for simplifying the boundary conditions, which would otherwise be awkward to impose. Therefore, in the present model, a curvilinear coordinate system is adopted, where $x_{1}$ (the direction along which the wave propagates) is replaced by $\phi$, the velocity potential of the wave motion, $x_{2}$ remains as the crossstream horizontal coordinate and $x_{3}$ (the vertical coordinate) is replaced by $\psi$, the streamfunction. The new curvilinear coordinates are defined by the relations

$$
U_{1}=\frac{\partial \phi}{\partial x_{1}}=\frac{\partial \psi}{\partial x_{3}}, \quad U_{3}=\frac{\partial \phi}{\partial x_{3}}=-\frac{\partial \psi}{\partial x_{1}}
$$

The spatial coordinates and components of vectors in the new curvilinear system retain the subscripts 1, 2 and 3, respectively, for the direction along the streamlines, horizontally across the streamlines and along lines of constant potential, but are distinguished from their Cartesian counterparts by a tilde. The infinitesimal length element in the direction along the streamlines is $\tilde{U}_{1}^{-1} \mathrm{~d} \phi$, where $\tilde{U}_{1}=\left(U_{1}^{2}+U_{3}^{2}\right)^{\frac{1}{2}}$ and the length element along the lines of constant potential is $\tilde{U}_{1}^{-1} \mathrm{~d} \psi$. The spatial derivatives along the 3 new coordinate directions are then defined as

$$
\frac{\partial}{\partial \tilde{x}_{1}}=\tilde{U}_{1} \frac{\partial}{\partial \phi}, \quad \frac{\partial}{\partial \tilde{x}_{2}}=\frac{\partial}{\partial x_{2}}, \quad \frac{\partial}{\partial \tilde{x}_{3}}=\tilde{U}_{1} \frac{\partial}{\partial \psi}
$$

The flow configuration and coordinate systems for a surface wave propagating in the positive $x_{1}$ direction are presented schematically in figure 1. Note that the orientation of the curvilinear coordinates is approximately in the opposite direction to the Cartesian coordinates (except for $\left.\tilde{x}_{2}\right)$, with $\tilde{x}_{1}$ pointing to the left and $\tilde{x}_{3}$ pointing downwards.

In the curvilinear coordinate system, the linearised Cauchy equation takes a form analogous to (2.6), but the strain tensor is considerably simpler than when expressed in a Cartesian coordinate system, namely

$$
\frac{\partial \tilde{\boldsymbol{x}}}{\partial \tilde{\boldsymbol{a}}}=\left(\begin{array}{ccc}
\tilde{U}_{1} / \tilde{U}_{10} & 0 & \tilde{U}_{1} \partial \tau_{0} / \partial \tilde{a}_{3}-\tilde{U}_{10} \partial \tau / \partial \tilde{x}_{3} \\
0 & 1 & 0 \\
0 & 0 & \tilde{U}_{10} / \tilde{U}_{1}
\end{array}\right)
$$

where the subscript 0 denotes variables evaluated at the initial time, before any turbulence distor- 


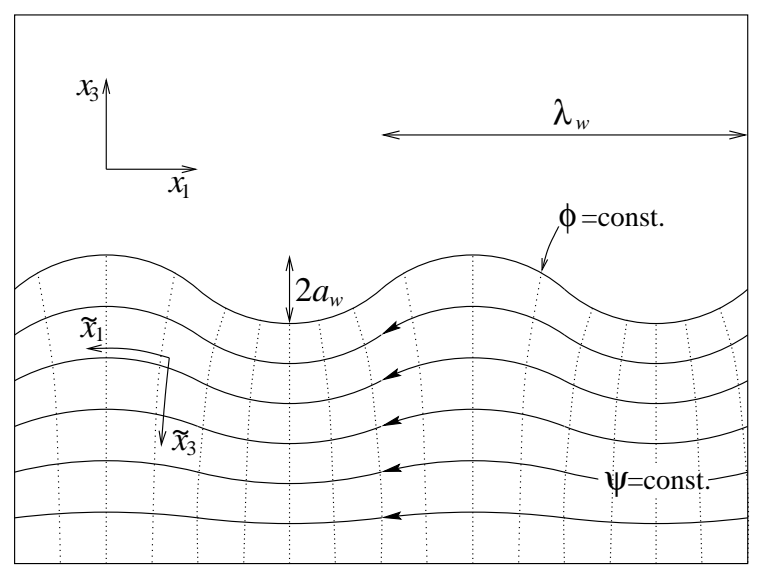

FIGURE 1. Schematic diagram of the model problem in a frame of reference travelling with the wave, showing the streamlines (solid) and lines of constant potential (dotted), and the Cartesian and curvilinear coordinate systems.

tion has taken place, and $\tau$ is the travel time of a fluid parcel, defined as

$$
\tau=\int^{\phi} \frac{\mathrm{d} \phi^{\prime}}{\tilde{U}_{1}^{2}} .
$$

For turbulence flowing around a bluff body the 'initial position', where the turbulence is undistorted, is infinitely upstream of the body, so $U_{10}$ is the free-stream velocity, which is assumed constant (Hunt 1973; Durbin 1981). As a consequence, $\partial \tau_{0} / \partial \tilde{a}_{3}=0$ and (2.11) simplifies further. If the mean flow is a periodic wave, however, there is no obvious choice for the initial position, which has to be imposed more arbitrarily. $\tilde{U}_{10}$ and $\partial \tau_{0} / \partial \tilde{a}_{3}$ then depend on the location of the initial position relative to the phase of the wave and must be retained in (2.11). This dependence of the model on the initial position will be explored in $\S 3$. In the case of turbulence generated by a breaking wave, it is perhaps to be expected that the turbulence is injected at the forward slope of the wave (Rapp \& Melville 1990), and as a result, most of the calculations presented in $\S 3$ use this as the initial position.

\subsection{The mean velocity field}

The mean velocity field considered in the present model is that associated with a relatively smallamplitude, monochromatic surface wave, expressed in a frame of reference travelling with the phase velocity of the wave $c_{w}=\sigma_{w} / k_{w}$. Following Longuet-Higgins (1984), the wave motion is expressed here as a function of the curvilinear coordinates $\phi$ and $\psi$, but only the first term in the corresponding series expansion (his equation (4.4)) is considered. This is a good approximation 
for low wave slopes. The horizontal and vertical velocity components are

$$
\begin{aligned}
& U_{1}=c_{w}\left(a_{w} k_{w} \mathrm{e}^{-k_{w} \psi / c_{w}} \cos \left(k_{w} \phi / c_{w}\right)-1\right) \\
& U_{3}=-c_{w} a_{w} k_{w} \mathrm{e}^{-k_{w} \psi / c_{w}} \sin \left(k_{w} \phi / c_{w}\right),
\end{aligned}
$$

which satisfy continuity and irrotationality, and lead to

$$
\tilde{U}_{1}=c_{w}\left(1+a_{w}^{2} k_{w}^{2} \mathrm{e}^{-2 k_{w} \psi / c_{w}}-2 a_{w} k_{w} \mathrm{e}^{-k_{w} \psi / c_{w}} \cos \left(k_{w} \phi / c_{w}\right)\right)^{\frac{1}{2}}
$$

The advantage of this peculiar formulation of the wave motion is that it enables an analytical evaluation of the travel time function $\tau$. On performing the integration over $\phi^{\prime}$ in (2.12), the result is

$$
\begin{aligned}
& \tau(\phi, \psi)=\frac{2}{c_{w} k_{w}} \frac{1}{1-a_{w}^{2} k_{w}^{2} \mathrm{e}^{-2 k_{w} \psi / c_{w}}}\left[\arctan \left(\frac{1+a_{w} k_{w} \mathrm{e}^{-k_{w} \psi / c_{w}}}{1-a_{w} k_{w} \mathrm{e}^{-k_{w} \psi / c_{w}}} \tan \left(\frac{k_{w} \phi}{2 c_{w}}\right)\right)\right. \\
& \left.+\pi \operatorname{Int}\left(\frac{k_{w} \phi / c_{w}+\pi}{2 \pi}\right)\right]+f(\psi),
\end{aligned}
$$

where $f$ is an arbitrary function and Int denotes 'integer part'. The second term between square brackets has to be introduced in order for $\tau$ to be a monotonically increasing function of the velocity potential, because the arctan function is limited to take values in the interval $(-\pi / 2, \pi / 2)$. It can be shown from $(2.15)$ that the travel time function is approximately equal to $\phi / c_{w}^{2}+f(\psi)$ for very low wave slopes, but deviates systematically towards higher values for larger $a_{w} k_{w}$. This is a manifestation of the Stokes drift of the wave. The connection between the Stokes drift of a surface wave and the so-called 'Darwin drift' (which is closely related to the travel time function) has been noted recently by Eames \& McIntyre (1999), although they did not calculate $\tau$ explicitly.

From (2.11), (2.14) and (2.15), it follows that the strain tensor is specified completely as a function of $\phi$ and $\psi, \phi_{0}$ and $\psi_{0}$. Therefore, to obtain the evolution of the turbulence along the wave profile, values for $\phi_{0}$ and $\psi_{0}$ must be chosen to specify the initial position, and then the relevant turbulent quantities may be calculated along a streamline $\left(\psi=\psi_{0}\right)$, for different values of $\phi$ (this is done below). However, it would be convenient to express the results as a function of more physically significant variables like time or space. A rigorous relation between the Cartesian and the curvilinear coordinates requires the numerical resolution of implicit equations, but a simple approximation, valid for low wave slopes, is readily available. Equations (2.9) and (2.13) show that, to zeroth order in the wave slope, $\phi \approx-c_{w} x_{1}$ and $\phi_{0} \approx-c_{w} a_{1}$. Now, to the same order of 
approximation, it follows from $(2.7)$ that $x_{1} \approx a_{1}-c_{w} t$. Then, subtracting the initial from the final potential, $\phi-\phi_{0}=-c_{w}\left(x_{1}-a_{1}\right)=c_{w}^{2} t$, so that finally

$$
t \approx\left(\phi-\phi_{0}\right) / c_{w}^{2}
$$

This shows that the Eulerian time can be approximately related to the potential function. When the turbulence statistics are plotted as a function of time, in $\S 3, t$ will always be defined according to (2.16), so it must be recalled, when interpreting the results, that (2.16) is only an approximate equality.

\subsection{Solution in terms of Fourier modes}

Far from the free surface, the turbulence is not affected by the wave nor directly by the boundary, and remains homogeneous, isotropic and stationary. Now, by assumption the scale over which the motion associated with the wave varies, $\lambda_{w}$, is much larger than the initial integral length scale of the turbulence (see the first equation of (2.3)), and so at distances from the free surface in the range $l<\tilde{x}_{3}<\lambda_{w}$, the turbulence is distorted by the wave motion but not directly by the boundary. In this region the turbulence is locally homogeneous, in the sense that it varies over a length scale that is much larger than its integral scale $l$. So, for depths greater than $l$, it is justified to represent the turbulent velocity as a three-dimensional Fourier integral, with space and time dependent wavenumbers that vary over the length scale $\lambda_{w}$, in order to account for the slight inhomogeneity of the mean flow. This is the slow-variation approximation, also used by Durbin (1981). In the curvilinear coordinate system, the turbulent velocity is thus

$$
\tilde{u}_{i}^{(H)}(\tilde{\boldsymbol{x}}, t)=\iiint \hat{\tilde{u}}_{i}^{(H)}(\tilde{\boldsymbol{k}}, \tilde{\boldsymbol{x}}, t) \mathrm{e}^{\mathrm{i} \tilde{\boldsymbol{k}} \cdot \tilde{\boldsymbol{x}}} \mathrm{d} \tilde{k}_{1} \mathrm{~d} \tilde{k}_{2} \mathrm{~d} \tilde{k}_{3}
$$

where $\tilde{\boldsymbol{k}}(\tilde{\boldsymbol{x}}, t)=\left(\tilde{k}_{1}, \tilde{k}_{2}, \tilde{k}_{3}\right)$ is the wavenumber vector, and the spatial coordinates in the plane of the wave motion can be approximated locally as $\tilde{x}_{1}=\phi / \tilde{U}_{1}, \tilde{x}_{3}=\psi / \tilde{U}_{1}$. The vorticity of the turbulence may be expressed in a formally similar way:

$$
\tilde{\omega}_{i}^{(H)}(\tilde{\boldsymbol{x}}, t)=\iiint \hat{\tilde{\omega}}_{i}^{(H)}(\tilde{\boldsymbol{k}}, \tilde{\boldsymbol{x}}, t) \mathrm{e}^{\mathrm{i} \tilde{\boldsymbol{k}} \cdot \tilde{\boldsymbol{x}}} \mathrm{d} \tilde{k}_{1} \mathrm{~d} \tilde{k}_{2} \mathrm{~d} \tilde{k}_{3}
$$

Invoking the slow-variation approximation, it is now possible to relate the Fourier amplitudes of the turbulent velocity and of the turbulent vorticity through an algebraic relation, in exactly the 
same way as in a Cartesian coordinate system, namely

$$
\hat{\tilde{\omega}}_{i}^{(H)}=\varepsilon_{i j k} \mathrm{i} \tilde{k}_{j} \hat{\tilde{u}}_{k}^{(H)}
$$

where terms of higher order in the parameter $\left(l / \lambda_{w}\right)$ have been ignored. Taking the external product of the wavenumber vector with this expression, an equation equivalent to (2.8) in the spectral domain is obtained,

$$
\hat{\tilde{u}}_{i}^{(H)}=\varepsilon_{i j k} \mathrm{i} \frac{\tilde{k}_{j}}{k^{2}} \hat{\tilde{\omega}}_{k}^{(H)}
$$

where $k=\left(\tilde{k}_{1}^{2}+\tilde{k}_{2}^{2}+\tilde{k}_{3}^{2}\right)^{\frac{1}{2}}$ is the wavenumber magnitude.

The Cauchy equation can be expressed in terms of Fourier amplitudes by using (2.6) (with tildes in the curvilinear coordinate system) and (2.18), yielding

$$
\begin{aligned}
& \hat{\tilde{\omega}}_{i}^{(H)}(\tilde{\boldsymbol{k}}, \tilde{\boldsymbol{x}}, t)=\frac{\partial \tilde{x}_{i}}{\partial \tilde{a}_{j}} \mathrm{e}^{\mathrm{i}\left(\tilde{\boldsymbol{k}}_{0} \cdot \tilde{\boldsymbol{a}}-\tilde{\boldsymbol{k}} \cdot \tilde{\boldsymbol{x}}\right)} \hat{\tilde{\omega}}_{j}^{(H)}\left(\tilde{\boldsymbol{k}}_{0}, \tilde{\boldsymbol{a}}, 0\right), \\
& \tilde{k}_{i}(\tilde{\boldsymbol{x}}, t)=\frac{\partial \tilde{a}_{j}}{\partial \tilde{x}_{i}} \tilde{k}_{0 j},
\end{aligned}
$$

where $\tilde{\boldsymbol{k}}_{0}=\left(\tilde{k}_{01}, \tilde{k}_{02}, \tilde{k}_{03}\right)=\tilde{\boldsymbol{k}}(\tilde{\boldsymbol{a}}, 0)$ is the wavenumber vector at the initial time. These two equations give the Lagrangian temporal evolution of, respectively, the Fourier amplitude of the turbulent vorticity and the wavenumber vector. Applying (2.19) to obtain the initial vorticity amplitude in (2.21) as a function of the velocity amplitude and inserting the final vorticity amplitude given by (2.21) into (2.20) yields

$$
\hat{\tilde{u}}_{i}^{(H)}(\tilde{\boldsymbol{k}}, \tilde{\boldsymbol{x}}, t)=-\varepsilon_{i j k} \varepsilon_{l m n} \frac{\tilde{k}_{j} \tilde{k}_{0 m}}{k^{2}} \frac{\partial \tilde{x}_{k}}{\partial \tilde{a}_{l}} \mathrm{e}^{\mathrm{i}\left(\tilde{\boldsymbol{k}}_{0} \cdot \tilde{\boldsymbol{a}}-\tilde{\boldsymbol{k}} \cdot \tilde{\boldsymbol{x}}\right)} \hat{\tilde{u}}_{n}^{(H)}\left(\tilde{\boldsymbol{k}}_{0}, \tilde{\boldsymbol{a}}, 0\right),
$$

which, together with (2.11), the second equation of (2.21) and (2.17), completely defines the final distorted turbulent velocity field as a function of the initial undistorted turbulent velocity field.

\subsection{Blocking effect of the boundary}

At distances from the free surface of $O(l)$ or shorter, the turbulence is no longer locally homogeneous, since it is forced to adjust to the boundary. For the turbulence with low Froude and Weber numbers considered here, the effect of the boundary is primarily blocking (Brocchini \& Peregrine 2000), so that the normal velocity component reduces to zero at the boundary. This effect is included in the model by adding an irrotational correction to the turbulent velocity field (Hunt \& Graham 1978). Equation (2.2) ensures that, for an irrotational mean flow, an initially irrotational 
velocity remains irrotational at all subsequent times. The total velocity field thus becomes

$$
\tilde{u}_{i}=\tilde{u}_{i}^{(H)}+\frac{\partial \phi^{(S)}}{\partial \tilde{x}_{i}}, \quad i=1,2,3,
$$

where $\phi^{(S)}(\tilde{\boldsymbol{x}}, t)$ is a velocity potential satisfying

$$
\nabla^{2} \phi^{(S)}=0
$$

subject to boundary conditions that ensure that the turbulent velocity is zero at the boundary and vanishes far from it:

$$
\frac{\partial \phi^{(S)}}{\partial \tilde{x}_{3}}\left(\tilde{x}_{3}=0\right)=-\tilde{u}_{3}^{(H)}\left(\tilde{x}_{3}=0\right) \quad \text { and } \quad \phi^{(S)}\left(\tilde{x}_{3} \rightarrow \infty\right)=0 .
$$

Although the velocity potential $\phi^{(S)}$ decays rapidly to zero for $\tilde{x}_{3}>l$, it remains locally homogeneous along the other two coordinate directions, so it can be expressed as a two-dimensional Fourier integral as follows:

$$
\phi^{(S)}(\tilde{\boldsymbol{x}}, t)=\iint \hat{\phi}^{(S)}\left(\tilde{k}_{1}, \tilde{k}_{2}, \tilde{\boldsymbol{x}}, t\right) \mathrm{e}^{\mathrm{i}\left(\tilde{k}_{1} \tilde{x}_{1}+\tilde{k}_{2} \tilde{x}_{2}\right)} \mathrm{d} \tilde{k}_{1} \mathrm{~d} \tilde{k}_{2}
$$

Invoking again the slow-variation approximation, the solution of (2.24) which can be expressed in the form (2.26) and satisfies the boundary conditions (2.25), is formally identical to that found by Hunt \& Graham (1978) for turbulence near a flat wall, namely

$$
\hat{\phi}^{(S)}\left(\tilde{k}_{1}, \tilde{k}_{2}, \tilde{\boldsymbol{x}}, t\right)=\int \frac{\hat{\tilde{u}}_{3}^{(H)}(\tilde{\boldsymbol{k}}, \tilde{\boldsymbol{x}}, t)}{\tilde{k}_{12}} \mathrm{e}^{-\tilde{k}_{12} \tilde{x}_{3}} \mathrm{~d} \tilde{k}_{3}
$$

where $\tilde{k}_{12}=\left(\tilde{k}_{1}^{2}+\tilde{k}_{2}^{2}\right)^{\frac{1}{2}}$. This expression differs only from the solution obtained by by Hunt \& Graham in that the turbulence is not perfectly homogeneous along the $\tilde{x}_{1}$ and $\tilde{x}_{2}$ directions, because the straining by the wave motion varies with $\tilde{x}_{1}$ and $\tilde{x}_{2}$, so that both the wavenumber $\tilde{\boldsymbol{k}}$ and $\hat{\tilde{u}}_{3}^{(H)}$ vary slowly in space and time.

\subsection{Complete solution}

From $(2.17),(2.23)$ and $(2.26)$, it follows that the total turbulent velocity field must be given by a two-dimensional Fourier integral in the form

$$
\tilde{u}_{i}(\tilde{\boldsymbol{x}}, t)=\iint \hat{\tilde{u}}_{i}\left(\tilde{k}_{1}, \tilde{k}_{2}, \tilde{\boldsymbol{x}}, t\right) \mathrm{e}^{\mathrm{i}\left(\tilde{k}_{1} \tilde{x}_{1}+\tilde{k}_{2} \tilde{x}_{2}\right)} \mathrm{d} \tilde{k}_{1} \mathrm{~d} \tilde{k}_{2}
$$

where the Fourier amplitude is defined by

$$
\hat{\tilde{u}}_{i}=\int \hat{\tilde{u}}_{i}^{(H)} \mathrm{e}^{\mathrm{i} \tilde{k}_{3} \tilde{x}_{3}} \mathrm{~d} \tilde{k}_{3}+\mathrm{i} \tilde{k}_{i} \hat{\phi}^{(S)}, \quad i=1,2
$$




$$
\hat{\tilde{u}}_{3}=\int \hat{\tilde{u}}_{3}^{(H)} \mathrm{e}^{\mathrm{i} \tilde{k}_{3} \tilde{x}_{3}} \mathrm{~d} \tilde{k}_{3}+\frac{\partial \hat{\phi}^{(S)}}{\partial \tilde{x}_{3}} .
$$

Taking into account (2.22), (2.27) and (2.29), the Fourier amplitude of the total distorted turbulent velocity (including the effects of distortion by the wave and by the boundary) may be related to the initial undistorted amplitude through

$$
\hat{\tilde{u}}_{i}\left(\tilde{k}_{1}, \tilde{k}_{2}, \tilde{\boldsymbol{x}}, t\right)=\int \tilde{M}_{i j}(\tilde{\boldsymbol{k}}, \tilde{\boldsymbol{x}}, t) \hat{\tilde{u}}_{j}^{(H)}\left(\tilde{\boldsymbol{k}}_{0}, \tilde{\boldsymbol{a}}, 0\right) \mathrm{d} \tilde{k}_{3},
$$

where the elements of the matrix $\tilde{M}_{i j}$ can be seen as 'transfer functions' (Hunt 1973). As was just seen, the blocking effect of the boundary may be taken into account by applying the changes due to blocking to the wave-distorted turbulence. Hence the functions $\tilde{M}_{i j}$ may be decomposed as

$$
\tilde{M}_{i j}(\tilde{\boldsymbol{k}}, \tilde{\boldsymbol{x}}, t)=\tilde{B}_{i k}(\tilde{\boldsymbol{k}}, \tilde{\boldsymbol{x}}, t) \tilde{W}_{k j}(\tilde{\boldsymbol{k}}, \tilde{\boldsymbol{x}}, t)
$$

where the matrix $\tilde{B}_{i k}$ accounts for blocking and the matrix $\tilde{W}_{i k}$ accounts for distortion by the wave.

The effect of the wave is deduced from (2.22), and leads to

$$
\tilde{W}_{i n}=-\varepsilon_{i j k} \varepsilon_{l m n} \frac{\tilde{k}_{j} \tilde{k}_{0 m}}{k^{2}} \frac{\partial \tilde{x}_{k}}{\partial \tilde{a}_{l}} \mathrm{e}^{\mathrm{i}\left(\tilde{\boldsymbol{k}}_{0} \cdot \tilde{\boldsymbol{a}}-\tilde{\boldsymbol{k}} \cdot \tilde{\boldsymbol{x}}\right)},
$$

while the effect of blocking is deduced from (2.27) and (2.29), yielding

$$
\begin{aligned}
& \tilde{B}_{i i}=\mathrm{e}^{\mathrm{i} \tilde{k}_{3} \tilde{x}_{3}}, \\
& \tilde{B}_{i 3}=\mathrm{i} \frac{\tilde{k}_{i}}{\tilde{k}_{12}} \mathrm{e}^{-\tilde{k}_{12} \tilde{x}_{3}}, \quad i=1,2, \\
& \tilde{B}_{33}=\mathrm{e}^{\mathrm{i} \tilde{k}_{3} \tilde{x}_{3}}-\mathrm{e}^{-\tilde{k}_{12} \tilde{x}_{3}},
\end{aligned}
$$

with the remaining elements of $\tilde{B}_{i j}$ being equal to zero.

The turbulent velocity distorted by both the wave orbital motion and by the boundary is thus completely defined as a function of the undistorted turbulent velocity. It remains to prescribe the characteristics of the undistorted turbulence and to calculate statistics of the turbulence at various stages of distortion.

\subsection{Statistics of the turbulent velocity field}

In order to analyse the structure of the turbulence, statistics of the turbulent velocity field are required. The intensity and correlation of the velocity fluctuations are characterised by the Reynolds 
stresses. These may be calculated from the Fourier amplitudes of the turbulent velocity using

$$
\overline{\tilde{u}_{i} \tilde{u}_{j}}=\iiint \int \overline{\hat{\tilde{u}}_{i}^{*}\left(\tilde{k}_{1}, \tilde{k}_{2}\right) \hat{\tilde{u}}_{j}\left(\tilde{k}_{1}^{\prime}, \tilde{k}_{2}^{\prime}\right)} \mathrm{d} \tilde{k}_{1} \mathrm{~d} \tilde{k}_{2} \mathrm{~d} \tilde{k}_{1}^{\prime} \mathrm{d} \tilde{k}_{2}^{\prime}
$$

where the asterisk denotes complex conjugation and the overbar denotes ensemble averaging. Equation (2.30) implies that

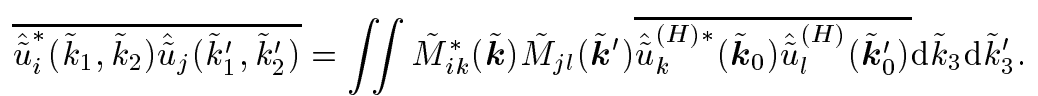

Now, the three-dimensional spectrum of the initial undistorted turbulent velocity, $\tilde{\Phi}_{i j}^{(H)}$, is defined as

$$
\overline{\hat{\tilde{\tilde{u}}}_{i}^{(H) *}\left(\tilde{\boldsymbol{k}}_{0}\right) \hat{\tilde{\hat{u}}}_{j}^{(H)}\left(\tilde{\boldsymbol{k}}_{0}^{\prime}\right)}=\tilde{\boldsymbol{\Phi}}_{i j}^{(H)}\left(\tilde{\boldsymbol{k}}_{0}\right) \delta\left(\tilde{\boldsymbol{k}}_{0}-\tilde{\boldsymbol{k}}_{0}^{\prime}\right),
$$

hence (2.34) and (2.35) can be used to obtain a simplified expression for the Reynolds stresses:

$$
\overline{\tilde{u}_{i} \tilde{u}_{j}}=\iiint \tilde{M}_{i k}^{*} \tilde{M}_{j l} \tilde{\Phi}_{k l}^{(H)} \mathrm{d} \tilde{k}_{1} \mathrm{~d} \tilde{k}_{2} \mathrm{~d} \tilde{k}_{3}
$$

The spatial structure of the turbulent velocity fluctuations is characterised by the integral length scales of the turbulence. For the velocity fluctuations $\tilde{u}_{i}$ and $\tilde{u}_{j}$ and along the direction $\tilde{x}_{l}$ these are defined by

$$
\tilde{L}_{i j}^{(l)}=\pi \frac{\tilde{\Theta}_{i j}^{(l)}\left(\tilde{k}_{l}=0\right)}{\overline{\tilde{u}_{i} \tilde{u}_{j}}}
$$

where $\tilde{\Theta}_{i j}^{(l)}$ is the one-dimensional wavenumber spectrum, along the $\tilde{x}_{l}$ direction, of the velocity fluctuations $\tilde{u}_{i}$ and $\tilde{u}_{j}$. Hence $\tilde{L}_{i j}^{(l)}$ is interpreted as the length over which the $\tilde{u}_{i}$ and the $\tilde{u}_{j}$ velocity fluctuations are correlated in the direction $\tilde{x}_{l}$. The one-dimensional spectrum along $\tilde{x}_{1}$ is defined in terms of $\tilde{\Phi}_{k l}^{(H)}$ as

$$
\tilde{\Theta}_{i j}^{(1)}\left(\tilde{k}_{1}, \tilde{x}_{3}, t\right)=\iint \tilde{M}_{i k}^{*} \tilde{M}_{j l} \tilde{\Phi}_{k l}^{(H)} \mathrm{d} \tilde{k}_{2} \mathrm{~d} \tilde{k}_{3},
$$

and an analogous definition is valid for the spectrum along $\tilde{x}_{2}$.

The undistorted turbulence is assumed to be isotropic, so its three-dimensional spectrum is related to the energy spectrum in the following way:

$$
\tilde{\Phi}_{i j}^{(H)}\left(\tilde{\boldsymbol{k}}_{0}\right)=\left(\delta_{i j}-\frac{\tilde{k}_{0 i} \tilde{k}_{0 j}}{k_{0}^{2}}\right) \frac{E\left(k_{0}\right)}{4 \pi k_{0}^{2}},
$$

where $E\left(k_{0}\right)$ is the energy spectrum and $k_{0}=\left(\tilde{k}_{01}^{2}+\tilde{k}_{02}^{2}+\tilde{k}_{03}^{2}\right)^{\frac{1}{2}}$ is the initial wavenumber magnitude. Following Hunt \& Graham (1978), the well-known von Kármán energy spectrum, which mimics 
an inertial subrange at high wavenumbers, is adopted here,

$$
E\left(k_{0}\right)=u^{2} l \frac{g_{2}\left(k_{0} l\right)^{4}}{\left(g_{1}+\left(k_{0} l\right)^{2}\right)^{\frac{17}{6}}},
$$

where $g_{1}=0.558$ and $g_{2}=1.196$ are dimensionless constants. In this equation and hereafter, the length scale of the turbulence $l$ is defined as the initial longitudinal integral length scale of any velocity component, i.e. $l=\tilde{L}_{i i}^{(i)}(t=0)$, for $i=1,2,3$.

All the statistics derived in this subsection are expressed in the curvilinear coordinate system aligned with the streamlines. However, these can readily be compared with statistics measured in a Cartesian coordinate system, because the two coordinate systems are approximately equivalent for waves of low slope, and in fact coincide exactly at the wave crests and at the wave troughs.

\subsection{Important parameters}

There are seven basic variables controlling the behaviour of the present model: three of them are determined by the mean flow, in this case a surface wave. They are the amplitude $a_{w}$, wavenumber $k_{w}$ and phase velocity $c_{w}$ of the wave. Two further variables characterise the turbulence: the initial RMS turbulent velocity $u$ and the initial integral length scale $l$. The remaining two variables are introduced by the initial conditions and the duration of the interaction between the turbulence and the wave: they are, respectively, the initial position relative to the wave phase, $\tilde{a}_{1}$, which may be approximated as $\tilde{a}_{1} \approx \phi_{0} / c_{w}$, and time $t$, which as was seen in $\S 2.2$ is $t \approx\left(\phi-\phi_{0}\right) / c_{w}^{2}$. From these variables, it is possible to construct five independent dimensionless parameters:

$$
a_{w} k_{w}, \quad k_{w} \tilde{a}_{1}, \quad k_{w} c_{w} t=\sigma_{w} t, \quad u / c_{w}, \quad k_{w} l
$$

It turns out that the statistics of the velocity field do not depend on parameter $u / c_{w}$. This ratio only influences the speed of the distortion of the turbulence by the wave, which determines, for example, the pressure. So, in the following section, the sensitivity of the model results to the 4 remaining parameters will be tested. Graphs of the normalised Reynolds stresses $\overline{\tilde{u}_{i} \tilde{u}_{j}} / u^{2}$ and integral length scales $\tilde{L}_{i j}^{(k)} / l$ will be plotted as functions of $t / T$, where $T=2 \pi / \sigma_{w}$ is the wave period, for different values of $a_{w} k_{w}, k_{w} \tilde{a}_{1}$ and $k_{w} l$. A few profiles of the Reynolds stresses as a function of $\tilde{x}_{3} / l$ will also be presented, for chosen values of $a_{w} k_{w}, k_{w} \tilde{a}_{1}, k_{w} l$ and $t / T$. 


\section{Results}

Results for the distortion of turbulence during a single wave cycle will be shown in $\S 3.1$ and $\S 3.2$. In $\S 3.3$, the distortion of the turbulence by the Stokes drift of the wave will be addressed, with the presentation of results for the Reynolds stresses, turbulent kinetic energy and integral length scales of the turbulence. In both subsections, the blocking effect of the boundary is ignored. This would correspond in practice to taking measurements at a depth $\tilde{x}_{3} \approx l$. In $\S 3.4$, the blocking effect of the boundary is briefly examined, and found to lead to changes to the results similar to those predicted by Hunt \& Graham (1978). In $§ 3.5$, some results for turbulence distorted by a uniform shear are reproduced, and compared with the results obtained for turbulence distorted by a wave. Finally, in $\S 3.6$ and $\S 3.7$, scaling analyses of the energy equations are performed, in order to estimate the energy transfer taking place between the wave and the turbulence, and its effect on each component of the flow. This allows quantification of the wave attenuation process due to turbulence first identified by Phillips (1959).

\subsection{Turbulence modulation in a wave cycle}

Figures $2-5$ show graphs of the diagonal components of the Reynolds stress tensor (velocity variances), as a function of time normalised by the wave period, for different initial positions and different wave slopes. In figure 2 , the initial position is at the forward slope of the wave, in figure 3 , it is at the wave crest. In figure 4 , the initial position is at the backward slope of the wave and in figure 5 it is at the wave trough.

Figures 2-5 show that the modulation of the Reynolds stresses by the wave intensifies as the wave steepness increases, as would be expected. This modulation is approximately sinusoidal at the lowest slopes, but becomes more asymmetric as $a_{w} k_{w}$ increases. This is partly due to the curvilinear coordinate system used because $\phi$ varies faster at the wave troughs than at the wave crests and the definition of $t$ is based on $\phi$ (see (2.16)). For the highest slopes considered, the value of the Reynolds stresses does not repeat itself after one complete cycle. This is a manifestation of the irreversible part of the distortion, which is caused by the Stokes drift, and will be treated in $\S 3.3$.

The Reynolds stress tangential to the free surface in the streamwise direction, $\overline{\tilde{u}_{1}^{2}}$, attains a 

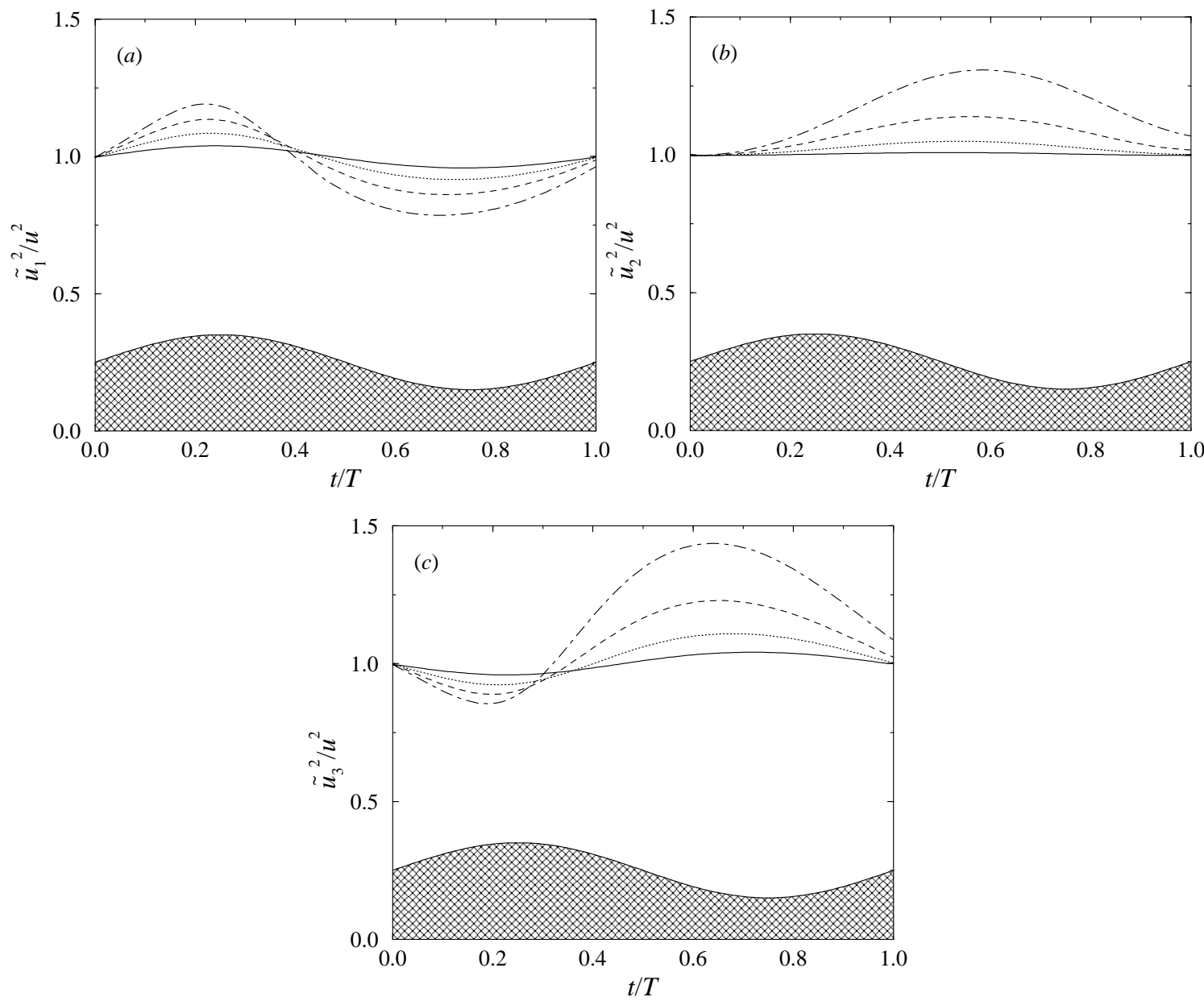

Figure 2. Modulation of the Reynolds stresses during a wave cycle, for turbulence initially at the forward slope of the wave, for $\tilde{x}_{3}=0, k_{w} l=0.6$. Solid line: $a_{w} k_{w}=0.05$, dotted line: $a_{w} k_{w}=0.1$, dashed line: $a_{w} k_{w}=0.15$, dash-dotted line: $a_{w} k_{w}=0.2$, hatched profile: shape of the distorting wave (arbitrary scale). (a) streamwise component, (b) spanwise component, (c) normal component.

maximum approximately at the wave crest and a minimum at the wave trough. The Reynolds stress normal to the free surface, $\overline{\tilde{u}_{3}^{2}}$, attains a maximum at the wave trough and a minimum at the wave crest. The tangential Reynolds stress in the cross-stream or spanwise direction, $\overline{\tilde{u}_{2}^{2}}$, first increases and then decreases, attaining a maximum approximately in the middle of the wave cycle, independent of the initial position.

Experimental and theoretical support for an increase in the intensity of the vertical velocity fluctuations and a decrease in the intensity of the streamwise velocity fluctuations at a hill crest (here equivalent to a wave trough) is provided by the work of Britter, Hunt \& Richards (1981) (their equation (3.3) and their figure 4b). Further experimental support for the predicted Reynolds 

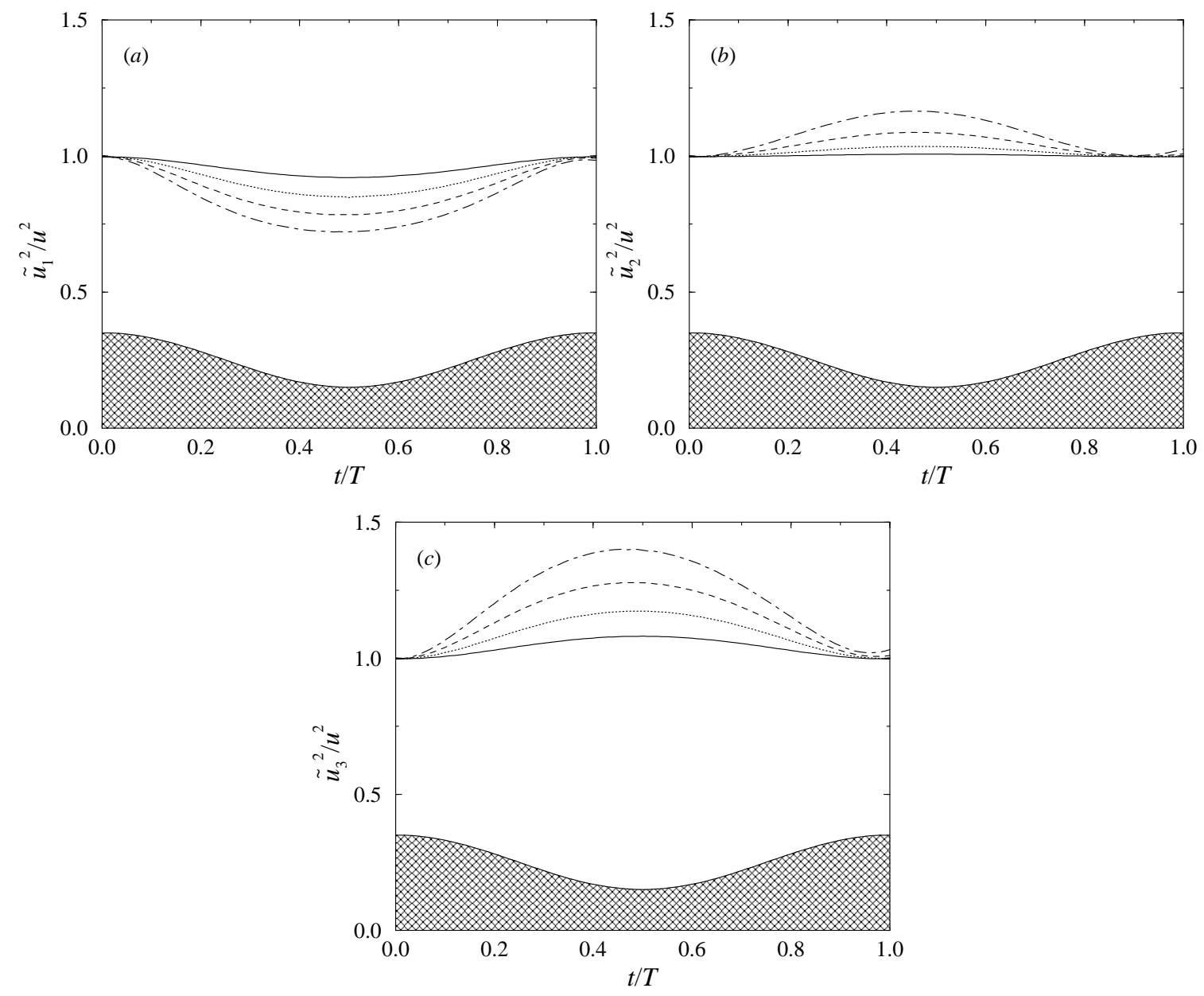

Figure 3 . Same as figure 2, but for turbulence initially at the wave crest.

stress modulation can be found in figure 10 of Thais \& Magnaudet (1996), where the streamwise Reynolds stress is greater than the vertical stress at the wave crest, while the reverse happens at the wave trough.

The modulation of the streamwise and normal Reynolds stresses has a peak-to-peak amplitude of $\approx 0.2$ for a wave slope of $a_{w} k_{w}=0.1$ and $\approx 0.4$ for $a_{w} k_{w}=0.2$, whereas the modulation of the spanwise Reynolds stress has smaller amplitude, perhaps $\approx 0.1$ for $a_{w} k_{w}=0.1$ and $\approx 0.2$ for $a_{w} k_{w}=0.2$. Although the data of Thais \& Magnaudet (1996) are affected by a stronger turbulence intensity at the wave trough, where the fixed probe almost touches the free-surface, it is possible to estimate the peak to peak modulations of the streamwise and vertical Reynolds stresses from their figure 10 by determining the value by which the streamwise stress exceeds the vertical stress at the wave crest and the vertical stress exceeds the streamwise stress at the wave trough. From visual 

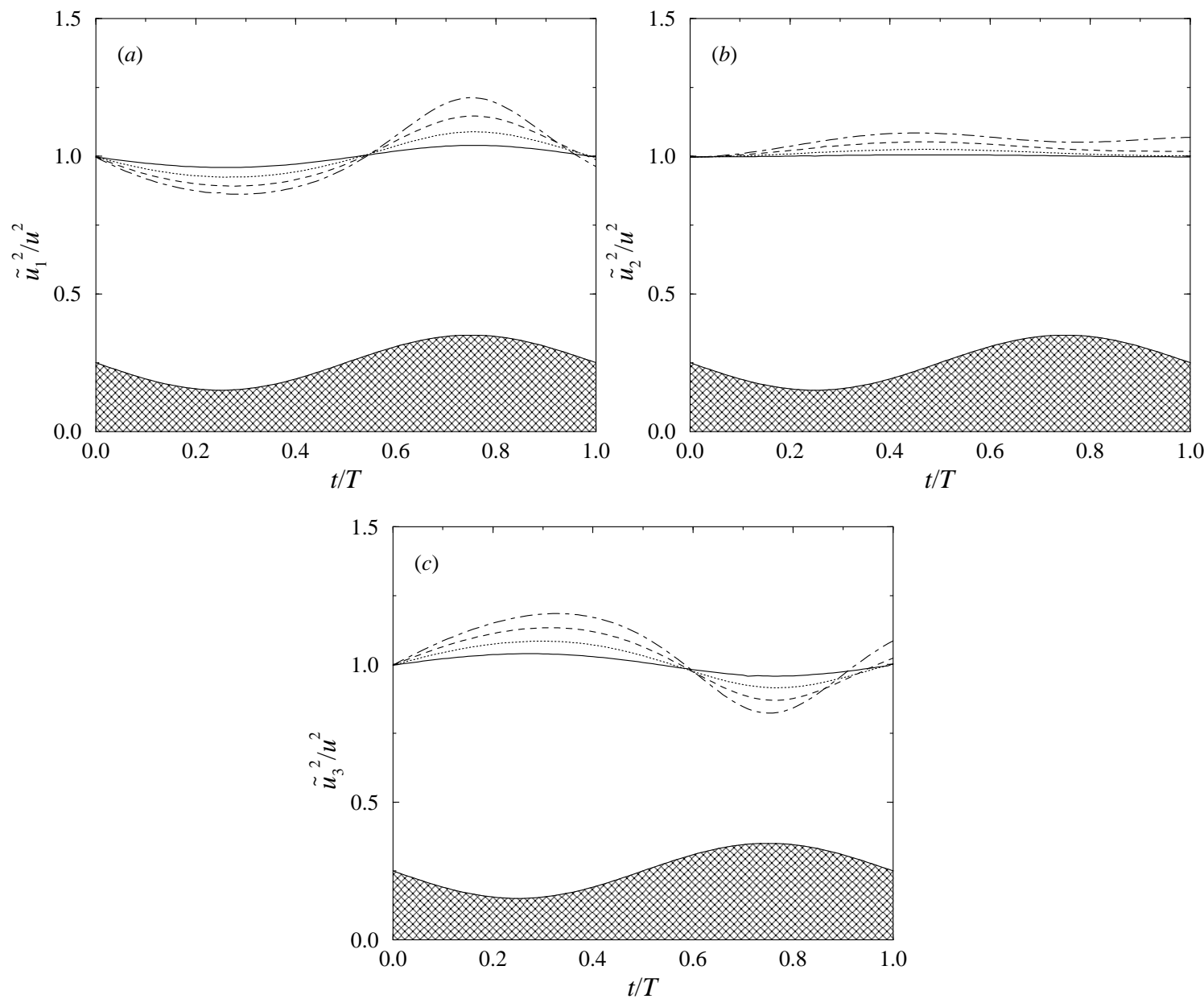

FiguRE 4. Same as figure 2, but for turbulence initially at the backward slope of the wave.

inspection, this is estimated as $\approx 0.2-0.3$, for a wave slope of $\approx 0.1$, and is therefore consistent with the present results. These results will now be explained using a simplified model.

\subsection{Simplified model for distortion over a wave cycle}

To first order in the wave slope, the distorting effect of a progressive surface wave on turbulence can be understood if the wave is described in a fixed Cartesian coordinate system, where the orbital motion can be written

$$
\begin{aligned}
& U_{1}\left(x_{1}, x_{3}\right)=c_{w} a_{w} k_{w} \mathrm{e}^{k_{w} x_{3}} \cos \left(k_{w} x_{1}-\sigma_{w} t\right), \\
& U_{3}\left(x_{1}, x_{3}\right)=c_{w} a_{w} k_{w} \mathrm{e}^{k_{w} x_{3}} \sin \left(k_{w} x_{1}-\sigma_{w} t\right)
\end{aligned}
$$

for a surface elevation

$$
\zeta=a_{w} \cos \left(k_{w} x_{1}-\sigma_{w} t\right)
$$



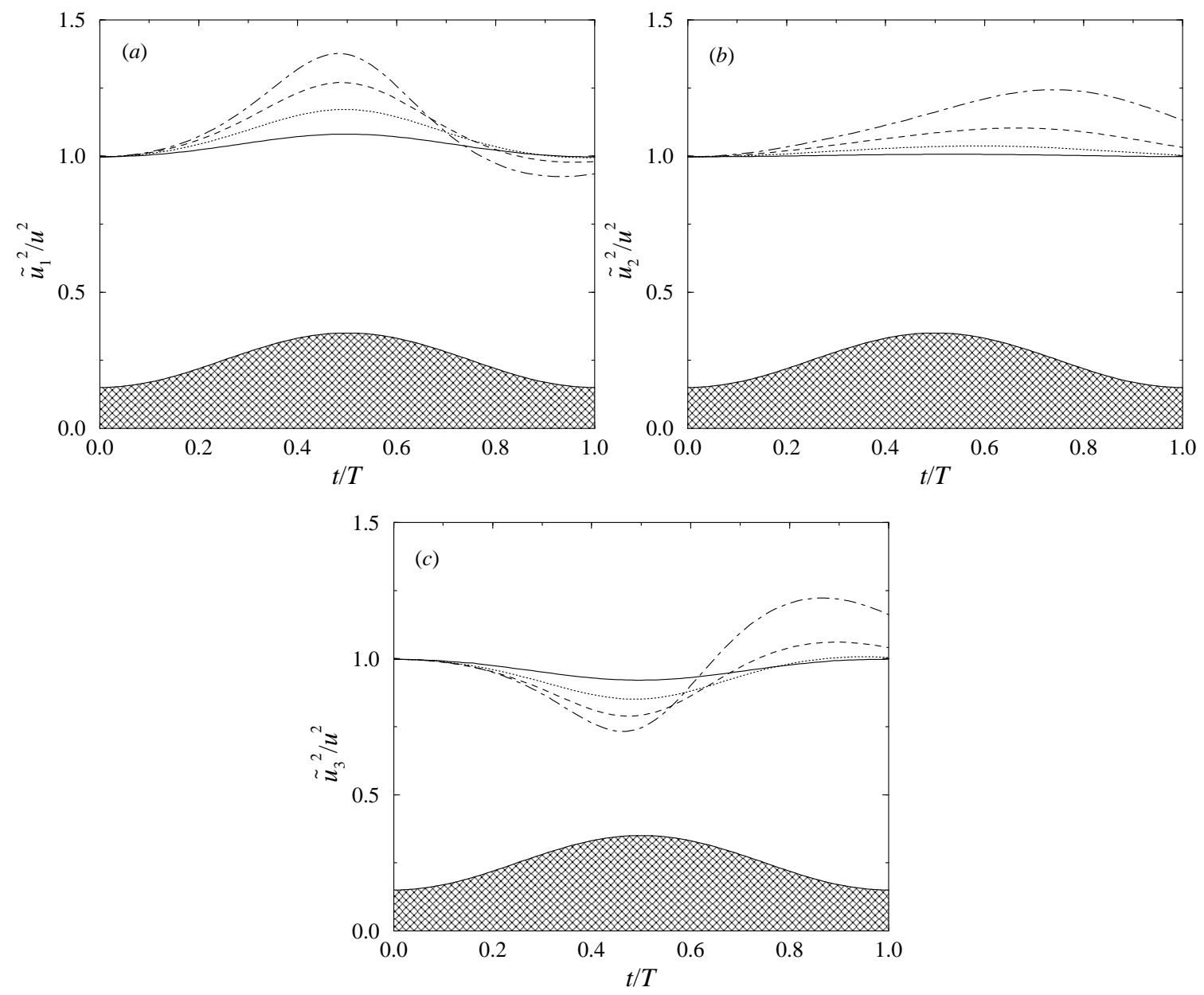

FiguRE 5. Same as figure 2, but for turbulence initially at the wave trough.

If (2.7) is differentiated with respect to the initial position, it is found that

$$
\frac{\partial x_{i}}{\partial a_{j}}=\delta_{i j}+\int_{0}^{t} \frac{\partial U_{i}}{\partial x_{k}} \frac{\partial x_{k}}{\partial a_{j}} \mathrm{~d} t^{\prime} .
$$

When the wave slope is sufficiently low, the distortion is relatively weak and the strain tensor inside the integral may be approximated as $\partial x_{k} / \partial a_{j} \approx \delta_{k j}$. On the other hand, the integration in time may be changed from an integration following the fluid parcels to a time integration at a fixed point. Then, differentiating the expressions (3.1) and inserting them into the integrals of (3.3), it is found after integration that, for small $a_{w} k_{w}$, the following expressions are approximately valid:

$$
\begin{aligned}
& \frac{\partial x_{1}}{\partial a_{1}}=1-a_{w} k_{w} \mathrm{e}^{k_{w} x_{3}}\left[\cos \left(k_{w} x_{1}-\sigma_{w} t\right)-\cos \left(k_{w} x_{1}\right)\right] \\
& \frac{\partial x_{1}}{\partial a_{3}}=\frac{\partial x_{3}}{\partial a_{1}}=-a_{w} k_{w} \mathrm{e}^{k_{w} x_{3}}\left[\sin \left(k_{w} x_{1}-\sigma_{w} t\right)-\sin \left(k_{w} x_{1}\right)\right] \\
& \frac{\partial x_{3}}{\partial a_{3}}=1+a_{w} k_{w} \mathrm{e}^{k_{w} x_{3}}\left[\cos \left(k_{w} x_{1}-\sigma_{w} t\right)-\cos \left(k_{w} x_{1}\right)\right]
\end{aligned}
$$




$$
\frac{\partial x_{1}}{\partial a_{2}}=\frac{\partial x_{2}}{\partial a_{1}}=\frac{\partial x_{2}}{\partial a_{3}}=\frac{\partial x_{3}}{\partial a_{2}}=0, \quad \frac{\partial x_{2}}{\partial a_{2}}=1 .
$$

These expressions show that $\partial x_{1} / \partial a_{1}$ is in phase opposition to the surface elevation and thus attains a maximum at the wave troughs and a minimum at the wave crests. This is best understood in the coordinate system travelling with the wave as being the result of the acceleration that the fluid suffers as it moves from crest to trough. $\partial x_{3} / \partial a_{3}$, on the other hand, is in phase with the surface elevation and attains a maximum at the crests and a minimum at the troughs. This result follows from the previous one by continuity: a fluid parcel that is stretched in one direction, must contract in the other.

The effect of this distortion on the vorticity is shown schematically in figure 6. At the wave crests, the fluid parcels are stretched vertically and compressed in the streamwise direction, leading to an intensification of the vertical vorticity and a weakening of the streamwise vorticity. Conversely, at the wave troughs the fluid parcels are stretched in the streamwise direction and compressed in the vertical, leading to an amplification of the streamwise vorticity and a weakening of the vertical vorticity.

The streamwise vorticity has contributions from the spanwise and vertical velocity components and the vertical vorticity has contributions from the streamwise and spanwise velocity. Therefore, at the wave crests, the streamwise turbulence intensity should increase and the vertical turbulence intensity should decrease, while at the troughs, the streamwise turbulence intensity should decrease and the vertical turbulence intensity should increase. This reasoning seems to explain the qualitative behaviour of $\overline{\tilde{u}_{1}^{2}}$ and $\overline{\tilde{u}_{3}^{2}}$ over one wave cycle.

It can also be seen from (3.4) that $\partial x_{1} / \partial a_{3}$ and $\partial x_{3} / \partial a_{1}$ are both out of phase by $\pi / 2$ relative to the surface elevation, attaining maxima at the backward slope of the wave and minima at the forward slope. These components of the strain tensor lead to additional irrotational tilting and stretching of vorticity, whose effects on the Reynolds stresses are not as obvious as those associated with the diagonal components.

The diagonal components of the strain tensor are the extensions suffered by the fluid in the 3 coordinate directions, defined as $e_{i}=\partial x_{i} / \partial a_{i}$. For a slab-symmetric straining flow in the $x_{1}-x_{3}$ plane with the principal axes aligned with the Cartesian coordinate system (Townsend 1976), these 
extensions have to satisfy

$$
e_{1}=\beta, \quad e_{2}=1, \quad e_{3}=\beta^{-1}
$$

due to continuity. In the present case, it follows from (3.4) that

$$
\beta \approx 1-a_{w} k_{w} \mathrm{e}^{k_{w} x_{3}}\left[\cos \left(k_{w} x_{1}-\sigma_{w} t\right)-\cos \left(k_{w} x_{1}\right)\right]
$$

It is then possible to use equation (3.11.9) of Townsend (1976) to estimate the magnitude of the Reynolds stress modulation attributable to the extensions in the wave velocity field. Townsend's expressions state that, for $\beta$ sufficiently close to 1 ,

$$
\begin{aligned}
& \frac{\overline{u_{1}^{2}}}{u^{2}}=1-\frac{4}{5} \frac{\beta-\beta^{-1}}{\beta+\beta^{-1}} \\
& \overline{u_{2}^{2}} \\
& \frac{u^{2}}{\overline{u_{3}^{2}}}=1+\frac{8}{35}\left(\beta-\beta^{-1}\right)^{2} \\
& \frac{u^{2}}{2}=\frac{4}{5} \frac{\beta-\beta^{-1}}{\beta+\beta^{-1}} .
\end{aligned}
$$

Substituting $\beta$ using (3.6) and truncating to the lowest order with respect to the perturbation (in this case $\left.a_{w} k_{w}\right),(3.7)$ becomes

$$
\begin{aligned}
& \frac{\overline{u_{1}^{2}}}{u^{2}}=1+\frac{4}{5} a_{w} k_{w} \mathrm{e}^{k_{w} x_{3}}\left[\cos \left(k_{w} x_{1}-\sigma_{w} t\right)-\cos \left(k_{w} x_{1}\right)\right] \\
& \frac{u_{2}^{2}}{\overline{u^{2}}}=1+\frac{32}{35} a_{w}^{2} k_{w}^{2} \mathrm{e}^{2 k_{w} x_{3}}\left[\cos \left(k_{w} x_{1}-\sigma_{w} t\right)-\cos \left(k_{w} x_{1}\right)\right]^{2} \\
& \frac{u_{3}^{2}}{u^{2}}=1-\frac{4}{5} a_{w} k_{w} \mathrm{e}^{k_{w} x_{3}}\left[\cos \left(k_{w} x_{1}-\sigma_{w} t\right)-\cos \left(k_{w} x_{1}\right)\right]
\end{aligned}
$$

This confirms that the existence of a maximum in the streamwise Reynolds stress at the crest and a maximum in the normal Reynolds stress at the trough are due primarily to the extension and compression of the fluid parcels. Equation (3.8) also explains why the spanwise Reynolds stress always takes values above one independent of the initial position relative to the wave phase.

It is clear from (3.8) that both $\overline{u_{1}^{2}} / u^{2}$ and $\overline{u_{3}^{2}} / u^{2}$ are predicted to undergo oscillations of peakto-peak amplitude $8 / 5\left(a_{w} k_{w}\right)$. For a wave slope of $a_{w} k_{w}=0.2$, this corresponds to $\approx 0.32$. On the other hand, $\overline{u_{2}^{2}} / u^{2}$ is predicted to undergo oscillations of peak-to-peak amplitude $32 / 35\left(a_{w} k_{w}\right)^{2}$ or $128 / 35\left(a_{w} k_{w}\right)^{2}$ depending on the initial position relative to the wave phase. For a wave slope of 0.2 , this corresponds to $\approx 0.04$ and 0.15 respectively. These results are roughly consistent with what is observed in figures $2-5$.

The differences between the predictions of this simplified model and those from the full model 


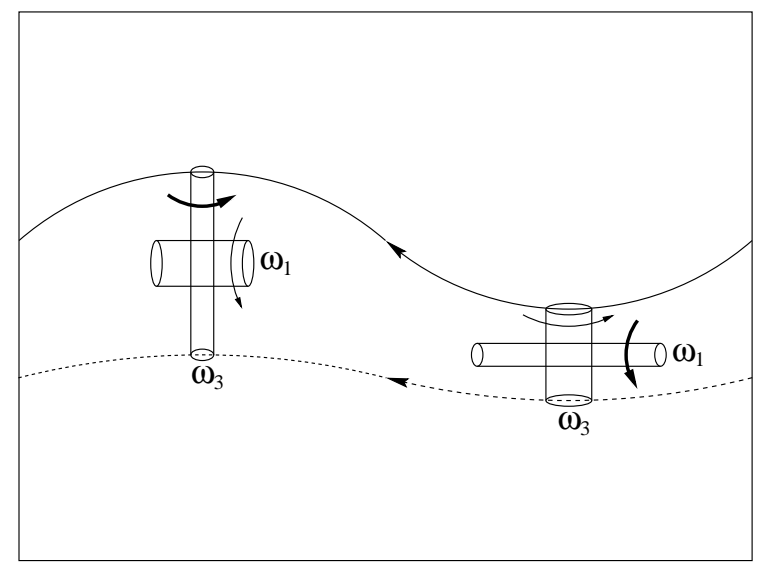

FIGURE 6. Schematic diagram showing the vorticity stretching and compression induced by the orbital motion at the crest and at the trough of a surface wave, in a frame of reference travelling with the wave.

are due to two factors: the neglect of the non-diagonal components of the strain tensor at all wave slopes, and the neglect of the Stokes drift, particularly at the higher slopes.

Equation (3.8) also helps to understand how the behaviour of the Reynolds stresses depends on the initial conditions. Due to the way in which the streamwise and normal Reynolds stresses are modulated, when the initial position is, for example, at a crest (figure $3 a$ ), the streamwise Reynolds stress departs from a maximum value, which can not be exceeded during its oscillation, whereas the normal Reynolds stress departs from a minimum (figure $3 c$ ). This corresponds to setting $k_{w} x_{1}=0$ in (3.8), which implies indeed that $\overline{u_{1}^{2}} / u^{2}$ is never larger than 1 and $\overline{u_{3}^{2}} / u^{2}$ is never smaller than 1 . When the initial position is at a trough $\left(k_{w} x_{1}=\pi\right)$, exactly the reverse occurs, as can be confirmed in figures $5(a, c)$.

Given the assumption of initial isotropy of the RDT model, it would seem that the most 'natural' initial positions are those in between crests and troughs, because at those positions both the streamwise and normal Reynolds stresses are in the middle of their oscillations, and the flow appears as little distorted as possible. However, the same is still not true for the spanwise Reynolds stress, $\overline{u_{2}^{2}}$, since this stress always departs from a minimum. The problem, which is clear inspecting (3.4), is that there is no region in a monochromatic surface wave where the flow can be considered naturally undistorted. Any possible choice of initial position $k_{w} x_{1}$ in (3.4) leads either the diagonal or the off-diagonal components of the strain tensor to oscillate asymmetrically with respect to the initial state. 


\subsection{Effect of the Stokes drift}

The distortion of the turbulence by the Stokes drift becomes clear after a considerable number of wave cycles.

Figure 7 shows the evolution of the diagonal components of the Reynolds stress tensor during 10 wave cycles, for a wave slope of 0.2 and different initial positions. The streamwise Reynolds stress, $\overline{\tilde{u}_{1}^{2}}$, attains maxima at the wave crests; the normal Reynolds stress, $\overline{\tilde{u}_{3}^{2}}$, attains maxima at

the wave troughs. The spanwise Reynolds stress, $\overline{\tilde{u}_{2}^{2}}$, always increases initially irrespective of the phase relation to the wave, as observed in figures $2(b), 3(b), 4(b)$ and $5(b)$. After a few periods it becomes phase-locked to the normal stress, attaining maxima at the wave troughs.

More importantly, the magnitude of the streamwise Reynolds stress progressively decreases, while the magnitudes of both the spanwise and the normal Reynolds stress progressively increase at a common rate. For the slope considered $\left(a_{w} k_{w}=0.2\right), \overline{\tilde{u}_{1}^{2}}$ becomes approximately half of its initial value after 10 wave cycles, while $\overline{\tilde{u}_{2}^{2}}$ and $\overline{\tilde{u}_{3}^{2}}$ increase by a factor of about 4 . This means that the turbulence becomes much more intense in the directions perpendicular to the direction of wave propagation. In other words: the turbulence becomes dominated by vortices with their axes of rotation aligned with the streamwise direction, as is the case in Langmuir circulations.

These results should be compared with those presented in figure 6 of McWilliams et al. (1997), from large-eddy simulations (LES) of turbulent flow in the ocean surface layer. Their figure shows profiles of the Reynolds stresses for turbulence in a shear current (without the effect of a Stokes drift) and when both shear and a Stokes drift are present, with the Stokes drift presumably having the dominant role (Langmuir turbulence). In the case of Langmuir turbulence, the spanwise and normal components of the Reynolds stress are distinctly larger than the streamwise component. This is consistent with the results of figure 7 .

The physical mechanism for the intensification of the streamwise vortices in the present model is the same as mechanism CL2 of Craik \& Leibovich (1976) for the generation of Langmuir circulations. It involves the tilting of vertical vorticity by the Stokes drift of the wave and its amplification as streamwise vorticity (figure 8). The difference is that the Craik-Leibovich formulation departs from an infinitesimal vertical vorticity perturbation arising from transverse variations of 

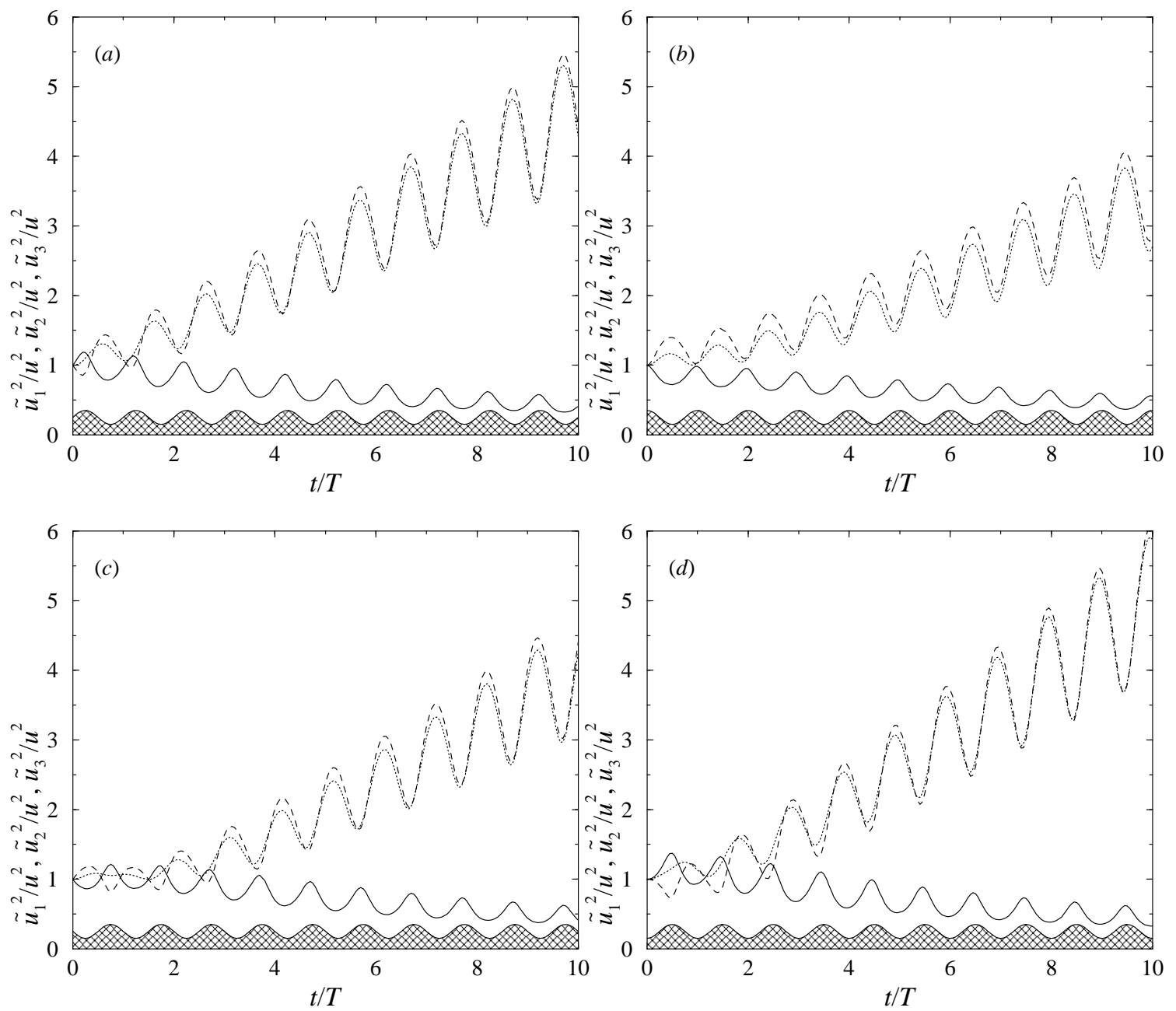

Figure 7. Evolution of the Reynolds stresses over 10 wave cycles, for $\tilde{x}_{3}=0, k_{w} l=0.6, a_{w} k_{w}=0.2$. Solid line: streamwise component, dotted line: spanwise component, dashed line: normal component, hatched profile: shape of the distorting wave (arbitrary scale). Turbulence initially at: (a) forward slope of wave, (b) wave crest, (c) backward slope of wave, $(d)$ wave trough.

the wind-induced shear current, whereas in the present model, there is initially a finite and isotropic distribution of vorticity, associated with the turbulence. In both cases, the Stokes drift selectively amplifies the vertical vorticity component as streamwise vorticity.

Figure 9 shows the time evolution of the Reynolds shear stress, $\overline{\tilde{u}_{1} \tilde{u}_{3}}$, during 10 wave cycles, for the same conditions as figure 7 . Since the turbulence is initially isotropic, the shear stress is initially zero. However, as the turbulence evolves, the shear stress grows to a negative value, stabilising at $\approx-0.7 u^{2}$. Like the velocity variances, the shear stress also oscillates during a wave cycle. At initial stages in the turbulence evolution, shear stress maxima (in absolute value) coincide 


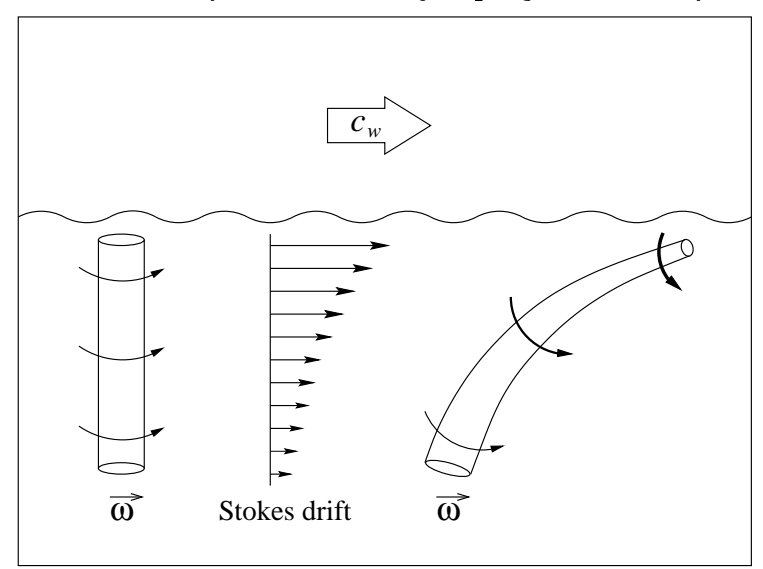

FIGURE 8. Schematic diagram showing the tilting and stretching of the vertical vorticity carried out by the Stokes drift of a surface wave over a number of wave cycles, in a fixed frame of reference.

with the backward slopes of the waves, and shear stress minima with the forward slopes. After 10 wave cycles, as the shear stress appears to attain a stable mean value, the maxima occur instead at the wave crests and the minima occur at the wave troughs.

Physically, the existence of a non-zero shear stress in the turbulence is due to the skewing of the velocity fluctuations carried out by the Stokes drift. As the vorticity is tilted from the vertical to an orientation sloping along the direction of wave propagation, it is at the same time amplified. Then, positive streamwise velocity fluctuations tend to be associated with negative normal velocity fluctuations, thereby making $\overline{\tilde{u}_{1} \tilde{u}_{3}}$ negative (see figure 8). The existence of a non-zero shear stress has important consequences for the energy balance of the turbulence, as will be seen in $\S 3.6$.

Figure 10 shows the time evolution of the turbulent kinetic energy (TKE), defined as $E_{K}=$ $1 / 2\left(\overline{\tilde{u}_{1}^{2}}+\overline{\tilde{u}_{2}^{2}}+\overline{\tilde{u}_{3}^{2}}\right)$, during 10 wave cycles, for the same conditions as figure 7 . It can be seen that the TKE is also modulated by the waves, displaying an oscillatory behaviour, and tends to become dominated by the spanwise and normal Reynolds stresses as time advances. The TKE progressively increases and attains a value approximately 3 times higher than initially after 10 wave cycles, again cf. McWilliams et al. (1997).

Figures $2-5$ have shown that the modulation of the turbulence in a wave cycle is sensitive to the initial conditions. Figures 7, 9 and 10 now show that not only the oscillatory behaviour but also the overall growth rate of the Reynolds stresses due to the Stokes drift depends on the initial conditions. For example, it is clear in figure 9 that the shear stress is largest when the initial 

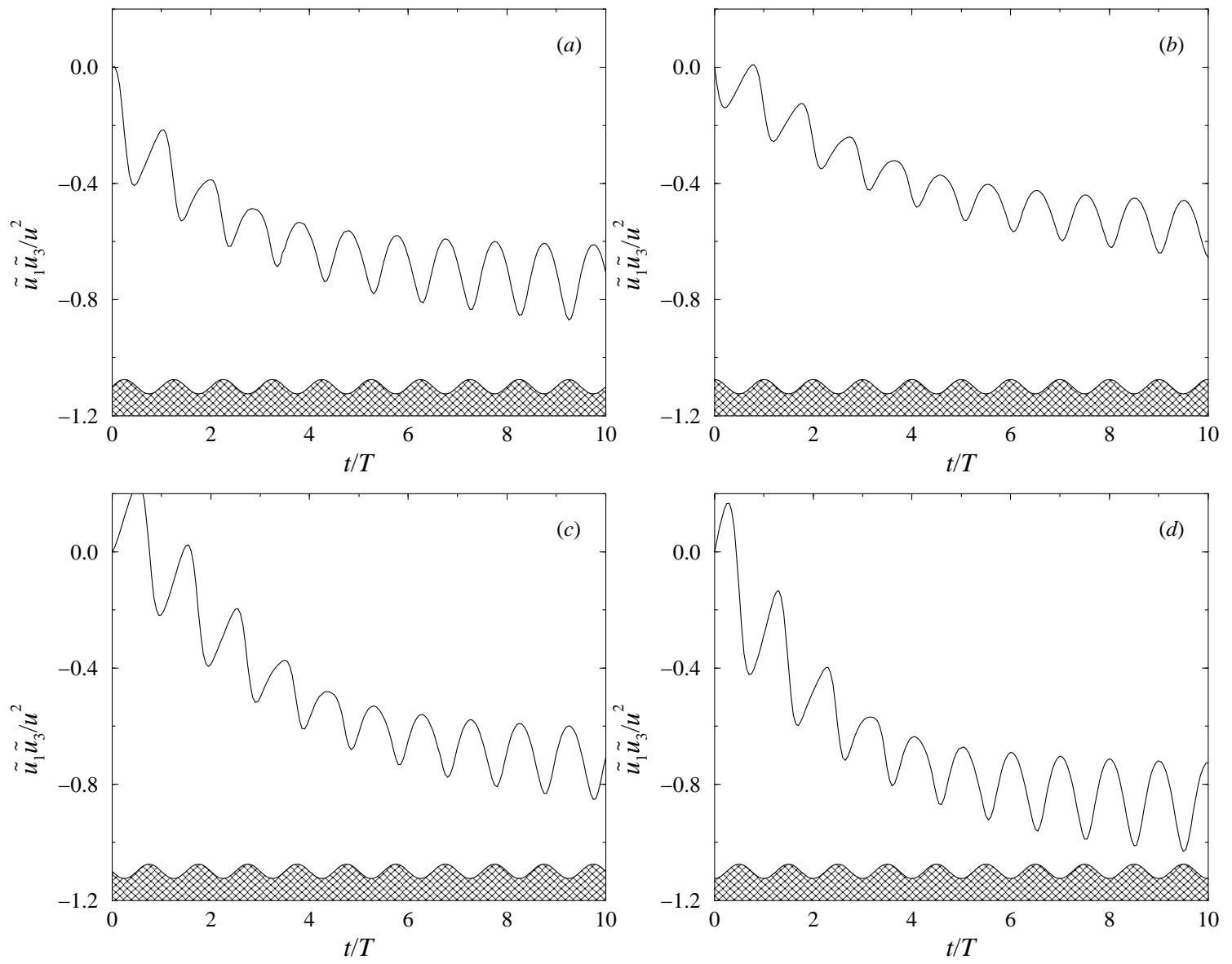

Figure 9. Evolution of the Reynolds stresses over 10 wave cycles, for $\tilde{x}_{3}=0, k_{w} l=0.6, a_{w} k_{w}=0.2$. Solid line: shear stress, hatched profile: shape of the distorting wave (arbitrary scale). Turbulence initially at: (a) forward slope of wave, $(b)$ wave crest, $(c)$ backward slope of wave, $(d)$ wave trough.

position is at the wave trough (figure $9 d$ ) and smallest when it is at the wave crest (figure $9 b$ ). Correspondingly, the TKE growth rate is fastest when the initial position is at the wave trough (figure $10 d$ ) and slowest when it is at the wave crest (figure 10b). The remaining plots of figures 9 and 10 show the same trend, suggesting a link between TKE growth and the shear stress. This link will be confirmed and further explored in $§ 3.6$.

The behaviour of the Reynolds stresses over several wave cycles is different for different initial conditions because the average values of the Reynolds stresses, over the first wave cycle, are also different. On a time scale longer than a wave cycle, varying the initial position of the turbulence relative to the wave phase is thus approximately equivalent to varying the initial turbulence intensity slightly. However, the importance of the initial conditions is limited, because, as was seen in $\S 3.2$, the fractional variation of the Reynolds stresses due to varying the initial position is, at most, 

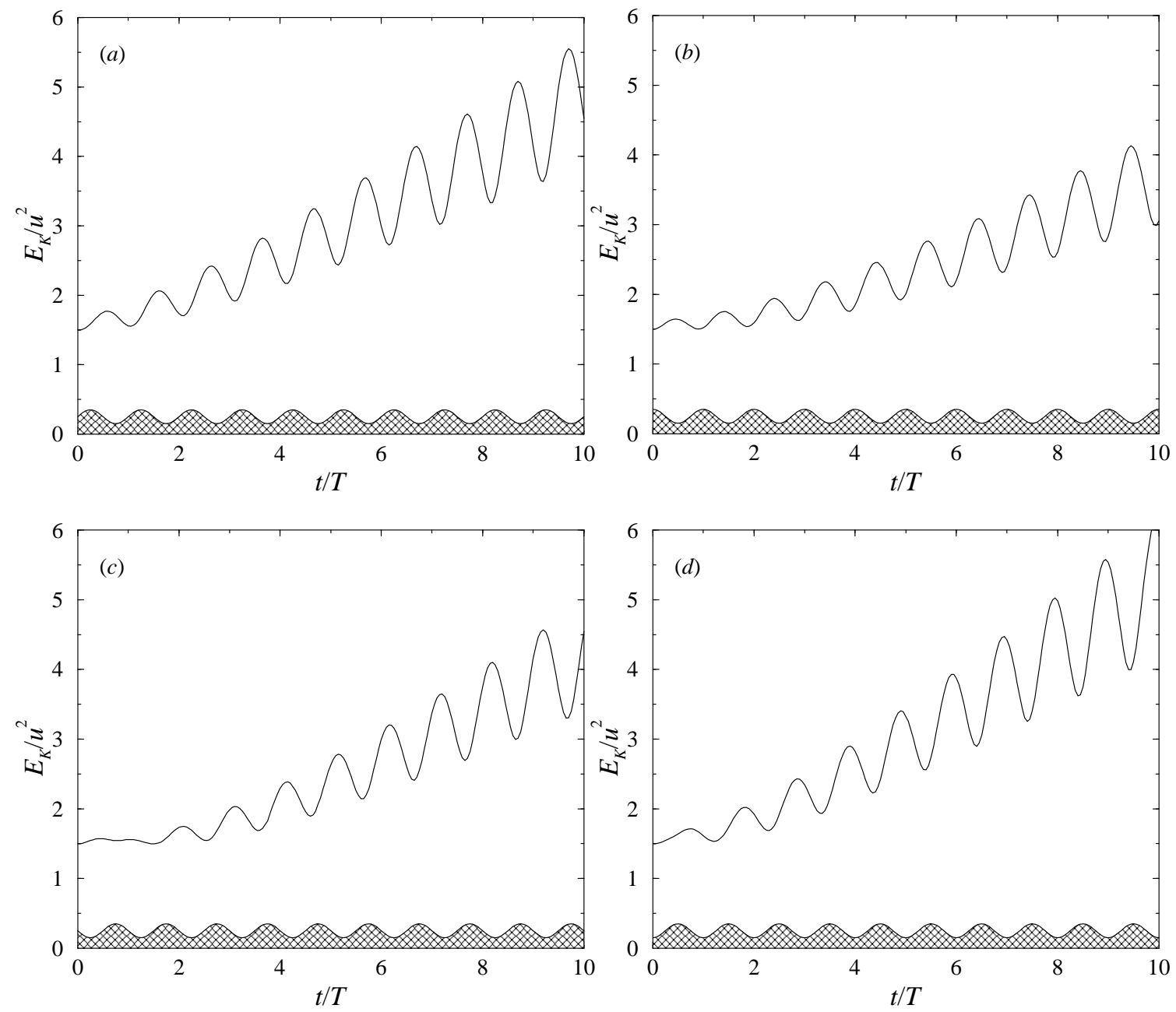

Figure 10. Evolution of the TKE over 10 wave cycles, for $\tilde{x}_{3}=0, k_{w} l=0.6, a_{w} k_{w}=0.2$. Solid line: turbulent kinetic energy, hatched profile: shape of the distorting wave (arbitrary scale). Turbulence initially at: (a) forward slope of wave, $(b)$ wave crest, $(c)$ backward slope of wave, $(d)$ wave trough.

of $O\left(a_{w} k_{w}\right)$, which is small. That explains why the four graphs of figures 7,9 and 10 resemble each other very much. For definiteness, all future results will consider an initial position at the forward slope of the wave.

The intensity of the turbulent velocity fluctuations has been characterised in detail for turbulence distorted by a surface wave. The spatial structure of the turbulent velocity fluctuations can now be characterised by the integral length scales of the turbulence. These length scales are modulated over a wave cycle, like the Reynolds stresses, but their evolution over several wave cycles is of greater interest.

Figure 11 presents the time evolution of the streamwise and spanwise integral length scales 

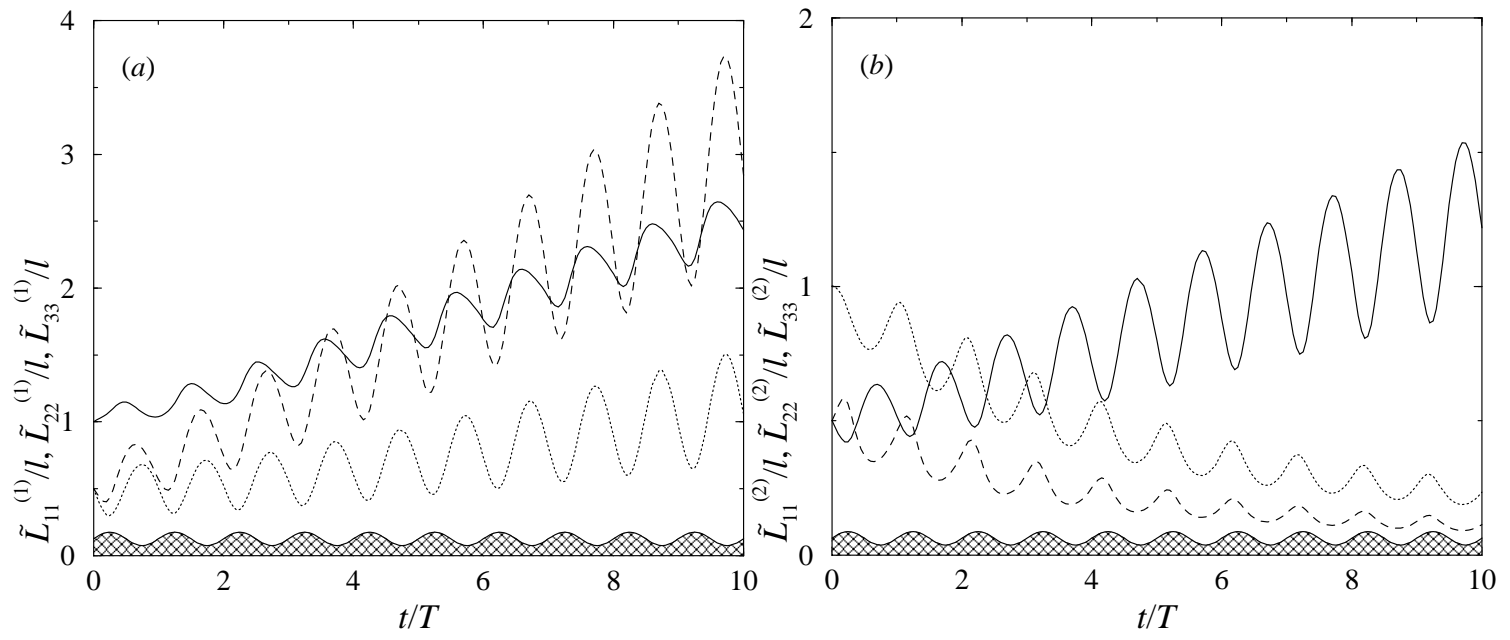

FiguRE 11. Evolution of the integral length scales over 10 wave cycles, for $\tilde{x}_{3}=0, k_{w} l=0.6, a_{w} k_{w}=0.2$. Hatched profile: shape of the distorting wave (arbitrary scale). (a) streamwise length scales. Solid line: $\tilde{L}_{11}^{(1)}$, dotted line: $\tilde{L}_{22}^{(1)}$, dashed line: $\tilde{L}_{33}^{(1)} \cdot(b)$ spanwise length scales. Solid line: $\tilde{L}_{11}^{(2)}$, dotted line: $\tilde{L}_{22}^{(2)}$, dashed line: $\tilde{L}_{33}^{(2)}$.

during 10 wave cycles, for a wave slope $a_{w} k_{w}=0.2$. First of all, it should be noted that, at $t=0$ the turbulence is isotropic, so the longitudinal integral length scales, $L_{11}^{(1)}$ and $L_{22}^{(2)}$, are 2 times larger than the transverse integral length scales, $L_{11}^{(2)}, L_{22}^{(1)}, L_{33}^{(1)}$ and $L_{33}^{(2)}$. This is typical of isotropic turbulence. Therefore the anisotropy of the turbulent flow structure can be evaluated by how much and in what way the integral length scales depart from these relative magnitudes.

In figure 11(a), it can be seen that all the streamwise length scales increase in time. The streamwise length scale of the $\tilde{u}_{1}$ velocity fluctuations, $\tilde{L}_{11}^{(1)}$, becomes $\approx 2.5$ times larger than initially after 10 wave cycles, while the corresponding amplification factors for the integral length scales of $\tilde{u}_{2}$ and $\tilde{u}_{3}, \tilde{L}_{22}^{(1)}$ and $\tilde{L}_{33}^{(1)}$, are $\approx 2$ and $\approx 6$, respectively. Figure $11(b)$ shows the time evolution of the spanwise integral length scales. It can be seen that only the integral length scale for the $\tilde{u}_{1}$ velocity component, $\tilde{L}_{11}^{(2)}$, increases in time, while the length scales for both $\tilde{u}_{2}$ and $\tilde{u}_{3}$, $\tilde{L}_{22}^{(2)}$ and $\tilde{L}_{33}^{(2)}$, decrease in time. After 10 wave cycles, $\tilde{L}_{11}^{(2)}, \tilde{L}_{22}^{(2)}$ and $\tilde{L}_{33}^{(2)}$ become respectively $\approx 2.5, \approx 0.25$ and $\approx 0.25$ times their initial values.

These results imply that the anisotropy of the streamwise velocity fluctuations remains small, whereas the spanwise and normal velocity fluctuations become elongated in the streamwise direction, with this elongation being especially pronounced for the normal velocity fluctuations. Hence, 
the streamwise vortices induced by the Stokes drift in the present model not only have their axes of rotation aligned with the streamwise direction (as shown by the Reynolds stresses), but they are also elongated in that direction, a feature which is commonly observed in Langmuir circulations (Faller \& Auer 1988).

These results should be compared with figures 12(b-d) of McWilliams et al. (1997), where horizontal cross-sections of the instantaneous velocity field near the surface are displayed. While, in these figures, the streamwise velocity fluctuations display no appreciable elongation in their structure, the spanwise and normal velocity components (shown in figures $12 b, c$ ) have contours that are clearly elongated in the streamwise direction, with this elongation being more pronounced for the normal velocity. This is consistent with the results of figure 11, and provides evidence that Langmuir turbulence in the simulations of McWilliams et al. (1997) resembles turbulence rapidly distorted by a surface wave in the present model.

The results presented until now have been calculated without taking into account the effect of blocking by the boundary on the turbulence. That effect will be considered briefly next.

\subsection{Blocking effect of the boundary}

As noted in $\S 2.4$, if at the initial time when the turbulence is undistorted by the wave, the blocking effect of the free surface is described by the theory of Hunt \& Graham (1978), this blocking effect remains purely kinematic at all subsequent times, and does not substantially alter the results obtained in the preceding subsections (which are essentially linked with vorticity distortion).

Since Hunt \& Graham's theory can be applied directly to the turbulence distorted by the wave, with the completely undistorted turbulence that formerly served as input being simply replaced by slowly varying turbulence, many of their conclusions remain valid, albeit with slight alterations. For example, the result which states that the TKE at the boundary has the same value as the TKE far from the boundary is now reformulated as

$$
\left(\overline{\tilde{u}_{1}^{2}}+\overline{\tilde{u}_{2}^{2}}\right)\left(\tilde{x}_{3}=0\right)=\left(\overline{\tilde{u}_{1}^{2}}+\overline{\tilde{u}_{2}^{2}}+\overline{\tilde{u}_{3}^{2}}\right)\left(\tilde{x}_{3} \rightarrow \infty\right), \quad \text { when } \quad l \rightarrow 0, \lambda_{w} \rightarrow \infty
$$

This means that the TKE value at the boundary taking blocking into account is equal to the TKE value that would exist at the boundary if there was no blocking or, alternatively, approximately equal to the TKE immediately outside the layer directly influenced by blocking. 


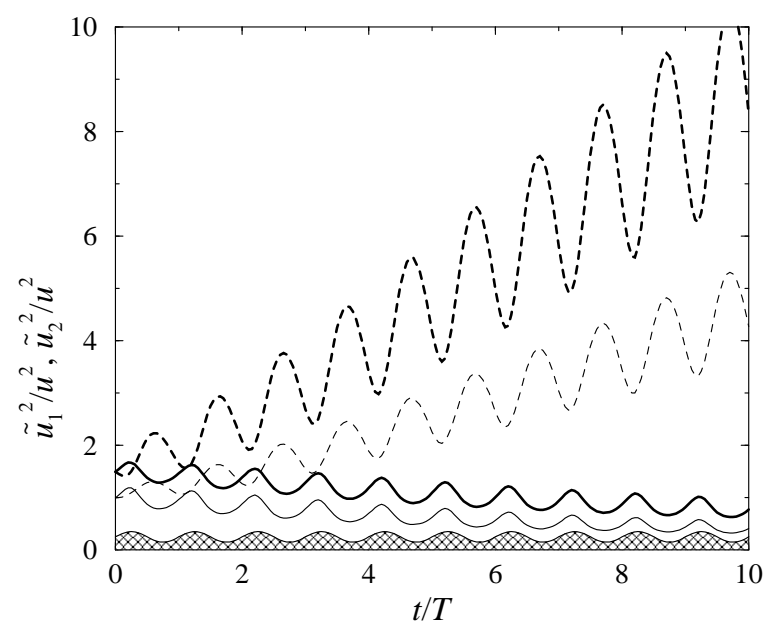

Figure 12. Evolution of the tangential Reynolds stresses over 10 wave cycles, with and without blocking, for $\tilde{x}_{3}=0, k_{w} l=0.6, a_{w} k_{w}=0.2$. Thick solid line: $\overline{\tilde{u}_{1}^{2}}$ with blocking, thin solid line: $\overline{\tilde{u}_{1}^{2}}$ without blocking, thick dashed line: $\overline{\tilde{u}_{2}^{2}}$ with blocking, thin dashed line: $\overline{\tilde{u}_{2}^{2}}$ without blocking, hatched profile: shape of the distorting wave (arbitrary scale).

Figure 12 shows the time evolution of the streamwise and spanwise Reynolds stresses, $\overline{\tilde{u}_{1}^{2}}$ and $\overline{\tilde{u}_{2}^{2}}$, during 10 wave cycles, with and without blocking. The curves relative to the blocked and non-blocked cases only differ in magnitude, and there are no appreciable differences in shape. The factor by which the curves with blocking exceed those without blocking increases from 1.5 at the initial time (as predicted by Hunt \& Graham 1978) to a higher value later. This is due to the fact that, in the blocked case, both $\overline{\tilde{u}_{2}^{2}}\left(l \ll \tilde{x}_{3} \ll \lambda_{w}\right)$ and $\overline{\tilde{u}_{3}^{2}}\left(l \ll \tilde{x}_{3} \ll \lambda_{w}\right)$ increase due to the Stokes drift, but only $\overline{\tilde{u}_{1}^{2}}\left(\tilde{x}_{3}=0\right)$ and $\overline{\tilde{u}_{2}^{2}}\left(\tilde{x}_{3}=0\right)$ are not zero at the boundary (of which $\overline{\tilde{u}_{1}^{2}}\left(\tilde{x}_{3}=0\right.$ ) decreases in time).

Figure 13 presents profiles of the Reynolds stresses with and without blocking, at $t / T=5$, for different values of the dimensionless wavenumber $k_{w} l$. It is found that $k_{w} l$ only influences the shape of the Reynolds stresses in between the surface and the region far from the surface, leading to a faster or slower decay of the profiles due to the distorting effect of the wave. Obviously, the longer the wavelength (i.e., the smaller $k_{w} l$ ), the deeper the distorting effect of the wave can penetrate. However, the value of the Reynolds stresses exactly at the boundary does not depend on $k_{w} l$, either when blocking is considered or when it is not. This justifies a posteriori why the parameter $k_{w} l$ has not been varied in previous tests.

Figures $13(a, b)$ show that, in the blocked case, $\overline{\tilde{u}_{1}^{2}}$ and $\overline{\tilde{u}_{2}^{2}}$ are amplified at the boundary by a 

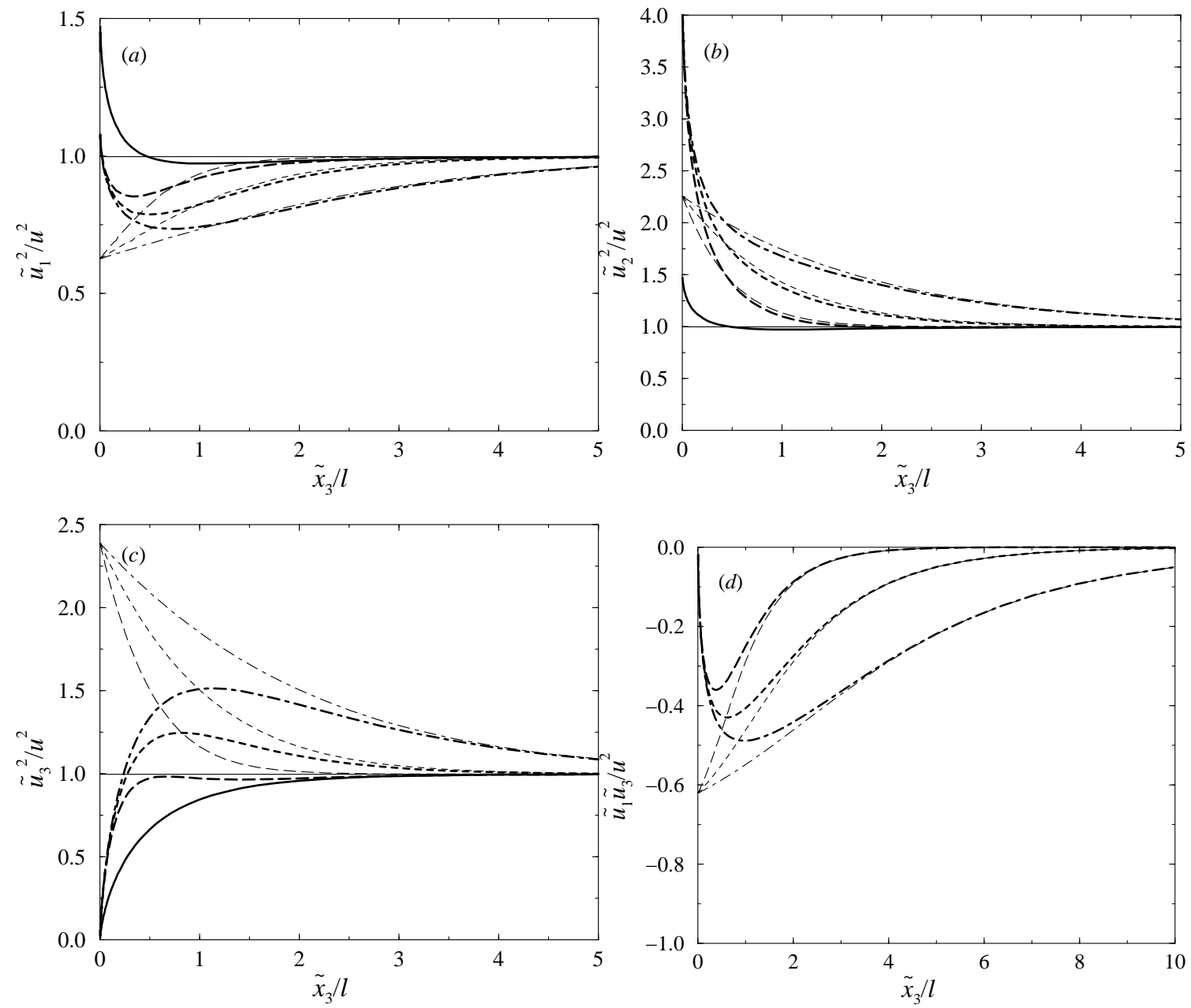

Figure 13. Profiles of the Reynolds stresses, with and without blocking, for $a_{w} k_{w}=0.2$. Thick lines: with blocking, thin lines: without blocking. Solid lines: at $t / T=0$, long-dashed lines: at $t / T=5$, with $k_{w} l=0.6$, dashed lines: at $t / T=5$ with $k_{w} l=0.3$, dash-dotted lines: at $t / T=5$ with $k_{w} l=0.15$. (a) streamwise component, $(b)$ spanwise component, $(c)$ normal component, $(d)$ shear stress.

factor greater than 1.5 relative to the unblocked case, consistent with figure 12 . The distortion caused by the wave counteracts this amplification in figure $13(a)$, so that $\overline{\tilde{u}_{1}^{2}}$ at the boundary is only slightly larger than far from the boundary, whereas in figure 13(b), the distortion caused by the wave reinforces the amplification of $\overline{\tilde{u}_{2}^{2}}$ due to blocking. In figure $13(c)$, it can be seen that $\overline{\tilde{u}_{3}^{2}}$ is forced to decay to zero towards the boundary over a length scale $l$, as expected, retaining nevertheless a value greater than 1 in the region $l \ll \tilde{x}_{3} \ll \lambda_{w}$. When blocking is considered, the shear stress, $\overline{\tilde{u}_{1} \tilde{u}_{3}}$, also has to decay to zero towards the boundary as $\tilde{u}_{3}$ decays to zero (figure $13 d)$. 
From a Lagrangian perspective, the shear current induced by the wind in the ocean surface layer and the Stokes drift of a wave appear at first to be rather similar, since both flows are characterised by a transport that has a maximum at the surface and decays with depth. These two types of flow might be expected to affect the turbulence in a similar way. However, their fundamental dynamics are quite different, as the numerical simulations of McWilliams et al. (1997) have made clear. One aim of the present section is to examine the extent to which linear processes that are accounted for in RDT explain these differences.

To a first approximation, the interaction between turbulence and a shear current can be understood by using a model similar to that formulated by Durbin (1978) as an extension of the original RDT model of Townsend (1970). This model incorporates the effects of a mean shear with a constant shear rate and of a rigid boundary, and assumes initially homogeneous and isotropic turbulence far from the boundary. Lee \& Hunt (1989) and Mann (1994) have shown that this type of model is able to describe qualitatively the turbulence structure in turbulent boundary layers. Detailed technical descriptions of the model can be found in Durbin (1978) and Mann (1994).

Durbin's model is used in this subsection to explain the differences between turbulence distortion by a shear current and turbulence distortion by a Stokes drift. Only the behaviour of the turbulence far from a boundary, which is assumed to exist at $x_{3}=0$, is examined, for a shear flow aligned with the $x_{1}$ direction, having a shear rate $\Gamma$.

Figure 14 shows the time evolution of the diagonal components of the Reynolds stress tensor induced by mean shear far from the boundary. This figure should be compared with figure 7 , which shows similar quantities (albeit in the curvilinear coordinate system) for turbulence distorted by a surface wave. The behaviour of the stresses differs markedly between the two cases. While in turbulence distorted by a wave, the streamwise stress decreases and the spanwise and normal stresses increase over a number of wave periods, in turbulence distorted by a shear the streamwise and spanwise stresses, $\overline{u_{1}^{2}}$ and $\overline{u_{2}^{2}}$, increase and the normal stress, $\overline{u_{3}^{2}}$, decreases. And while in turbulence distorted by a wave the spanwise and normal stresses become much larger than the streamwise stress, in turbulence distorted by a shear $\overline{u_{1}^{2}}$ becomes larger than $\overline{u_{2}^{2}}$, which in turn 


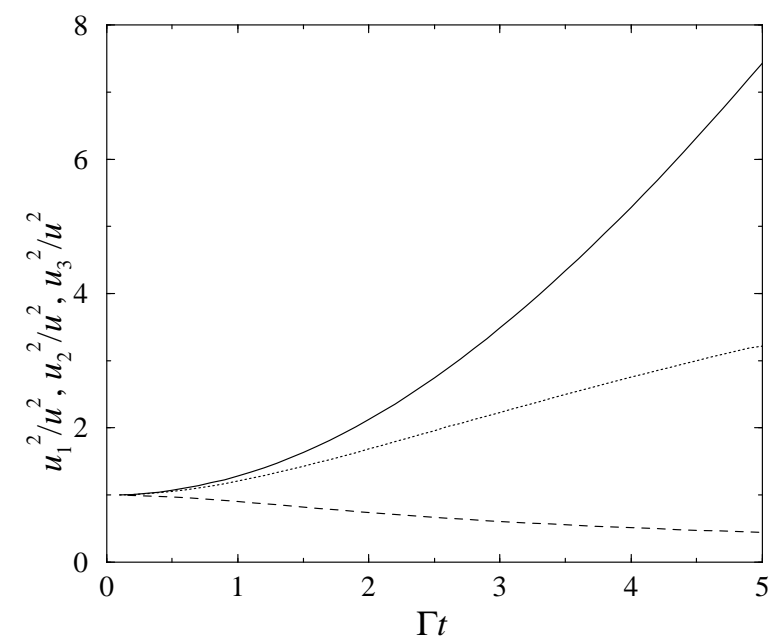

FiguRE 14. Evolution of the Reynolds stresses in a uniform shear flow, far from the boundary, as a function of dimensionless time $\Gamma t$. Solid line: streamwise component $\overline{u_{1}^{2}}$, dotted line: spanwise component $\overline{u_{2}^{2}}$, dashed line: normal component $\overline{u_{3}^{2}}$.

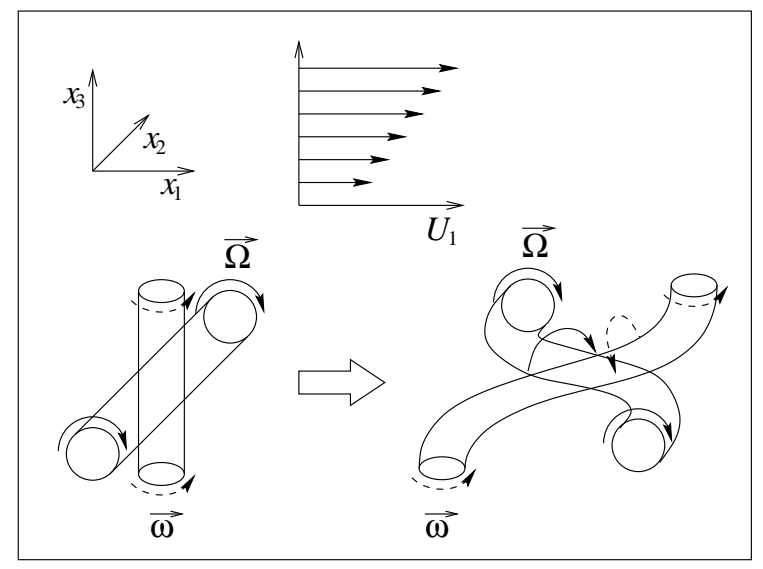

FIGURE 15. Schematic diagram showing the interaction between the mean and the turbulent vorticity in turbulence distorted by a shear flow. The induced circulations lead to a tilting of the mean vorticity and of the vertical turbulent vorticity that favour a partial cancellation of the spanwise and normal velocity fluctuations (on the right).

becomes larger than $\overline{u_{3}^{2}}$. Physically, this behaviour is due to the existence of vorticity in the shear flow, that does not exist in the Stokes drift. The circulations induced by the tilting of vorticity of the mean flow by the turbulence, in the case of the shear flow, counteract the circulations induced by the tilting of turbulent vorticity by the mean flow, so that $\overline{u_{2}^{2}}$ and $\overline{u_{3}^{2}}$ are prevented from becoming dominant (see figure 15).

Figure 14 should also be compared with figure 6 of McWilliams et al. (1997), where profiles of the Reynolds stresses in turbulence embedded in a shear current (without a Stokes drift) are denoted 
by the solid curves. These curves show that the streamwise stress is larger than the spanwise stress, which in turn is larger than the normal stress, near the surface. Hence, the present constant-shear model is able to explain the anisotropy of the turbulent velocity fluctuations in a shear current.

Figure 16 displays the time evolution of the integral length scales of turbulence distorted by a mean shear. Both the streamwise and the spanwise integral length scales of $u_{2}, L_{22}^{(1)}$ and $L_{22}^{(2)}$, decrease in time, and both the streamwise and the spanwise integral length scales of $u_{3}, L_{33}^{(1)}$ and $L_{33}^{(2)}$, increase in time. Hence the structure of these two velocity components remains approximately isotropic. However, the streamwise integral length scale of $u_{1}, L_{11}^{(1)}$, increases in time, while the spanwise length scale of the same velocity component, $L_{11}^{(2)}$, decreases in time. This means that the streamwise velocity fluctuations become elongated in the streamwise direction. Elongated structures in the streamwise turbulent velocity field are a well known feature of turbulent shear flows, where such structures are often called streaky structures (Kline et al. 1967). Good examples of streaky structures produced in a turbulent boundary layer by DNS can be found, for example, in figures 5, 7 and 9 of Lee et al. (1990), where horizontal cross-sections of the streamwise turbulent velocity are shown.

These results are in striking contrast with those presented for turbulence distorted by a surface wave: compare figure 16 with figure 11 . In figure 11 the structure of the $\tilde{u}_{2}$ and $\tilde{u}_{3}$ velocity fluctuations, as described by the integral length scales, becomes elongated, whereas in figure 16, it is the structure of $u_{1}$ that becomes elongated. Hence the present calculations explain the 2 basic flow regimes observed in the LES of McWilliams et al. (1997): shear turbulence and Langmuir turbulence. Although, in McWilliams et al.'s simulations of Langmuir turbulence, shear is also present, this shear appears to be sufficiently weak for the wave-turbulence interaction to dominate.

\subsection{Estimation of the turbulent kinetic energy growth}

In the preceding subsections, rigorous RDT calculations have been carried out. The final part of this paper is concerned instead with order-of-magnitude estimates relevant for the problems of streamwise vortex generation and surface wave decay. Nevertheless, the rigorous results obtained before will prove to be useful in guiding these estimates.

In $\S 3.3$, it was found that an increase in the TKE is predicted by the present model of turbulence 

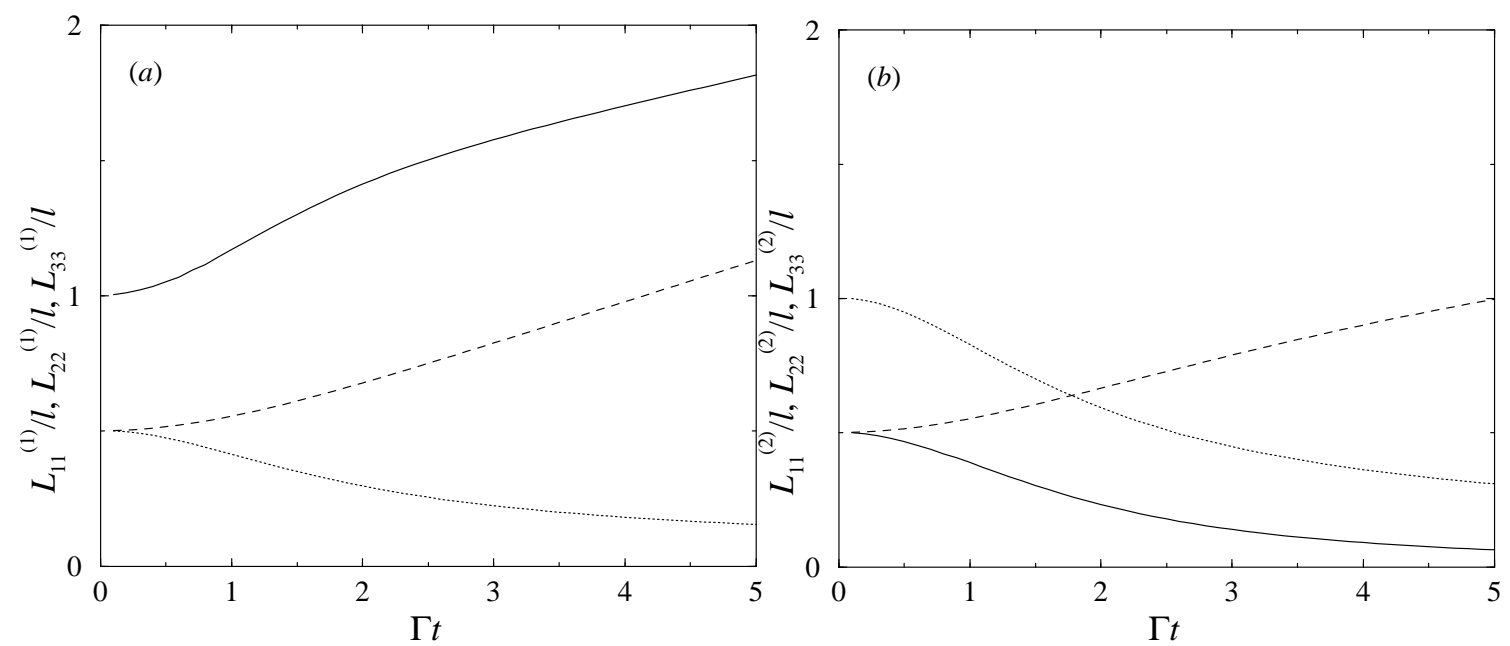

FigURE 16. Evolution of the integral length scales in a uniform shear flow, as a function of dimensionless time $\Gamma t$. (a) streamwise length scales. Solid line: $L_{11}^{(1)}$, dotted line: $L_{22}^{(1)}$, dashed line: $L_{33}^{(1)}$. $(b)$ spanwise length scales: Solid line: $L_{11}^{(2)}$, dotted line: $L_{22}^{(2)}$, dashed line: $L_{33}^{(2)}$.

distortion by a wave, which is related to the straining of the turbulence by the Stokes drift of the wave. In order to estimate this increase, it is necessary to derive an equation for the TKE compatible with the assumptions of the model. The TKE equation is here derived in a Cartesian coordinate system, for simplicity. But when the terms in that equation are estimated, the results of the preceding sections, which were found in the curvilinear coordinate system, will be used directly, since the behaviour of the statistics, in either coordinate system, is approximately equal.

The linearised momentum equation consistent with (2.2) is

$$
\frac{\partial u_{i}}{\partial t}+U_{j} \frac{\partial u_{i}}{\partial x_{j}}+u_{j} \frac{\partial U_{i}}{\partial x_{j}}=-\frac{1}{\rho} \frac{\partial p}{\partial x_{i}},
$$

where $\rho$ is the density and $p$ is the turbulent pressure. The required TKE equation may be obtained by multiplying (3.10) by $u_{i}$, adding all the expressions for $i=1,2,3$ and ensemble averaging. This yields

$$
\frac{\mathrm{d} E_{K}}{\mathrm{~d} t}=\left(\overline{u_{3}^{2}}-\overline{u_{1}^{2}}\right) \frac{\partial U_{1}}{\partial x_{1}}-2 \overline{u_{1} u_{3}} \frac{\partial U_{1}}{\partial x_{3}}-\frac{1}{\rho} \frac{\partial}{\partial x_{j}}\left(\overline{p u_{j}}\right) .
$$

The last term between square brackets in (3.11) appears in flux form and is associated with the redistribution of energy between different regions of the turbulent flow through pressure forces. In turbulence that is slowly varying in space, as considered here, this term has little importance, and so is neglected in the following. The first two terms on the right-hand side are production of turbulence by the mean flow, here the surface wave. It is the correlation between the wave strain 
rate and the Reynolds stresses in these terms that generates TKE. This correlation is calculated most naturally in a Lagrangian framework, that is, following the fluid parcels. Hence this effect can be estimated by studying the evolution of the TKE along a streamline.

Now, the material time derivative on the left-hand side of (3.11) is equivalent to a derivative with respect to the travel time $\tau$, which (2.12) shows is defined in terms of the velocity potential, and so in the frame of reference travelling with the wave, (3.11) may be expressed as

$$
\tilde{U}_{1}^{2} \frac{\partial E_{K}}{\partial \phi} \approx\left(\overline{u_{3}^{2}}-\overline{u_{1}^{2}}\right) \frac{\partial U_{1}}{\partial x_{1}}-2 \overline{u_{1} u_{3}} \frac{\partial U_{1}}{\partial x_{3}} .
$$

The Eulerian time, $t$, is related approximately to the wave potential by (2.16), and so the Eulerian time variation of the TKE in a fixed frame of reference can be written

$$
\frac{\partial E_{K}}{\partial t} \approx\left(\overline{u_{3}^{2}}-\overline{u_{1}^{2}}\right) \frac{c_{w}^{2}}{U_{1}^{2}+U_{3}^{2}} \frac{\partial U_{1}}{\partial x_{1}}-2 \overline{u_{1} u_{3}} \frac{c_{w}^{2}}{U_{1}^{2}+U_{3}^{2}} \frac{\partial U_{1}}{\partial x_{3}},
$$

where the definition of $\tilde{U}_{1}=\left(U_{1}^{2}+U_{3}^{2}\right)^{\frac{1}{2}}$ has been used.

Using (2.13) and (2.9), the wave strain rates in (3.13) become, to leading order in the wave slope,

$$
\begin{aligned}
& \frac{c_{w}^{2}}{U_{1}^{2}+U_{3}^{2}} \frac{\partial U_{1}}{\partial x_{1}} \approx \sigma_{w} a_{w} k_{w} \mathrm{e}^{-k_{w} \psi / c_{w}} \sin \left(k_{w} \phi / c_{w}\right) \\
& \frac{c_{w}^{2}}{U_{1}^{2}+U_{3}^{2}} \frac{\partial U_{1}}{\partial x_{3}} \approx \sigma_{w} a_{w} k_{w} \mathrm{e}^{-k_{w} \psi / c_{w}}\left[\cos \left(k_{w} \phi / c_{w}\right)+a_{w} k_{w} \mathrm{e}^{-k_{w} \psi / c_{w}}\right] .
\end{aligned}
$$

Both strain rates oscillate, the first with zero average and the second with positive average. Their time-averaged correlation with the oscillating Reynolds stress yields net TKE generation. The dominant contribution comes from $c_{w}^{2} /\left(U_{1}^{2}+U_{3}^{2}\right) \partial U_{1} / \partial x_{3}$. Its mean value can be estimated from the second contribution in (3.14), namely

$$
\frac{c_{w}^{2}}{U_{1}^{2}+U_{3}^{2}} \frac{\partial U_{1}}{\partial x_{3}} \approx\left(a_{w} k_{w}\right)^{2} \sigma_{w} \mathrm{e}^{-2 k_{w} \psi / c_{w}}=\frac{1}{2} \frac{\mathrm{d} u_{s}}{\mathrm{~d} x_{3}},
$$

where the Stokes drift velocity, $u_{s}$, is defined in an analogous way to the definition given in Cartesian coordinates, see equation (3.3.8) of Phillips (1977).

Now, the shear stress $\overline{u_{1} u_{3}}$ is negative, and so $-2 \overline{u_{1} u_{3}}\left(c_{w}^{2} / U_{1}^{2}+U_{3}^{2}\right) \partial U_{1} / \partial x_{3}$ generates TKE. Having in mind the estimates (3.14) and (3.15), the generation of TKE can be estimated by

$$
\frac{\partial E_{K}}{\partial t} \approx-2 \overline{u_{1} u_{3}} a_{w}^{2} k_{w}^{2} \sigma_{w} \approx-\overline{u_{1} u_{3}} \frac{\mathrm{d} u_{s}}{\mathrm{~d} x_{3}}\left(x_{3}=0\right) .
$$

This estimate of TKE production has a similar form to the term involving the Stokes drift in the 
TKE equation (5.1) of McWilliams et al. (1997). It is as if there is a Stokes drift 'shear' that generates TKE.

The quantitative accuracy of this reasoning is tested by comparing the TKE growth rate from this estimate with the growth rate calculated from the full RDT model. To compare this estimate more easily with the dimensionless growth rates available in figure 10, it should be noted that $\partial / \partial t=\left(\sigma_{w} / 2 \pi\right) \partial / \partial(t / T)$, hence $(3.16)$ becomes

$$
\frac{\partial E_{K}}{\partial(t / T)} \approx-4 \pi \frac{\overline{u_{1} u_{3}}}{u^{2}} a_{w}^{2} k_{w}^{2}
$$

Taking $a_{w} k_{w}=0.2$ and $-\overline{u_{1} u_{3}} / u^{2} \approx 0.7$, as suggested by the final portions of figure 9 , it follows that $\partial / \partial(t / T)\left(E_{K} / u^{2}\right)=0.35$. This is in remarkable agreement with the value that can be extracted directly by inspection from the slopes of the final portions of the curves in figure 10 . It thus appears that the estimates made above and the connection established between the TKE increase and the Reynolds shear stress are well founded.

To obtain an idea of the time-scales involved in the development of the streamwise vortices in the present model, a still rougher estimate may be carried out. Noting that $u_{i}=O(u)$ and $-\overline{u_{1} u_{3}}=O\left(u^{2}\right),(3.16)$ may be scaled as

$$
\frac{u^{2}}{T_{d}}=u^{2} a_{w}^{2} k_{w}^{2} \sigma_{w}
$$

where $T_{d}$ is the development time scale. With minor rearranging, (3.18) becomes

$$
T_{d}=\frac{1}{a_{w}^{2} k_{w}^{2} \sigma_{w}} .
$$

Taking reasonable values for the variables, like $a_{w} k_{w}=0.1$ and $\sigma_{w}=10 \mathrm{~s}^{-1}$, it is found that $T_{d}=10 \mathrm{~s}$. Hence the streamwise vortices that contain most of the TKE grow relatively fast.

\subsection{Estimation of turbulence-induced wave decay}

The previous results have established how the TKE of turbulence beneath a surface wave increases due to the distortion of the turbulence by the Stokes drift. Although in the RDT model developed here the turbulent flow has no feedback on the mean flow, which is taken as fixed, in real situations that is not the case. If a mean flow and a turbulent flow coexist in a fluid and the energy of the turbulent flow increases, that energy has to come from the mean flow, which correspondingly weakens. In the present case, the mean flow is associated with a surface wave, so the energy transfer 
taking place to the turbulence as the wave distorts the turbulence is necessarily linked with a decay of the wave. A mechanism of wave decay due to the straining of turbulence by the Stokes drift was first referred in the Introduction of Phillips (1959), who called it 'eddy-viscosity interaction'. Although Phillips (1959) did not establish a connection between the generation of streamwise vortices (which were in fact almost unknown at the time) and wave decay, that connection is implicit in his qualitative arguments involving vorticity stretching. It will be shown in this subsection that the energy transfer from the waves to the turbulence through this interaction can indeed account for the turbulence-induced wave decay observed in the experimental studies of Ölmez \& Milgram (1992) and Green et al. (1972).

Consider the momentum equation for the mean flow in a Cartesian coordinate system, now taking into account the Reynolds stresses:

$$
\frac{\partial U_{i}}{\partial t}+U_{j} \frac{\partial U_{i}}{\partial x_{j}}=-\frac{1}{\rho} \frac{\partial P}{\partial x_{i}}-\frac{\partial}{\partial x_{j}}\left(\overline{u_{i} u_{j}}\right)
$$

where $P$ is the mean pressure. If this equation is multiplied by $U_{i}$ and the resulting expressions for $i=1$ and 3 are added (noting that the $i=2$ component is zero for the monochromatic wave under consideration), an equation for the kinetic energy of the wave is obtained, namely

$$
\frac{\mathrm{d}}{\mathrm{d} t}\left(\frac{U_{1}^{2}+U_{3}^{2}}{2}\right)=\left(\overline{u_{1}^{2}}-\overline{u_{3}^{2}}\right) \frac{\partial U_{1}}{\partial x_{1}}+2 \overline{u_{1} u_{3}} \frac{\partial U_{1}}{\partial x_{3}}-\frac{\partial}{\partial x_{j}}\left(U_{k} \overline{u_{j} u_{k}}+\frac{1}{\rho} P U_{j}\right)
$$

The last term on the right-hand side appears in flux form and is related to transport processes, which do not change the total kinetic energy. The first two terms are identical to those found on the right-hand side of the TKE budget (3.11), albeit with with the opposite signs. Clearly these terms are associated with the energy transfer from the wave motion to the turbulent motion.

Since the growth rate of the TKE was estimated accurately assuming it to be solely determined by the production by 'Stokes drift shear', it is reasonable to estimate the decay of the kinetic energy of the wave by the same process, which yields

$$
\frac{\mathrm{d}}{\mathrm{d} t}\left(\frac{U_{1}^{2}+U_{3}^{2}}{2}\right) \approx \overline{u_{1} u_{3}} \frac{\mathrm{d} u_{s}}{\mathrm{~d} x_{3}}
$$

It remains to apply the same scaling ideas leading to (3.18) to the right-hand side of (3.22), and to note that the kinetic energy of the wave is $\left(U_{1}^{2}+U_{3}^{2}\right) / 2=O\left(a_{w}^{2} k_{w}^{2} c_{w}^{2}\right)$. Then, (3.22) may be 
scaled as

$$
\frac{\mathrm{d}}{\mathrm{d} t}\left(a_{w}^{2} k_{w}^{2} c_{w}^{2}\right) \approx-u^{2} a_{w}^{2} k_{w}^{2} \sigma_{w} \Rightarrow \frac{1}{a_{w}^{2}} \frac{\mathrm{d} a_{w}^{2}}{\mathrm{~d} t} \approx-\frac{u^{2}}{c_{w}^{2}} \sigma_{w}
$$

which implies that the wave amplitude decays exponentially in time due to the turbulence, with an attenuation rate

$$
\beta_{t}=a\left(\frac{u}{c_{w}}\right)^{2} \sigma_{w}
$$

where $a$ is a dimensionless constant of $O(1)$.

The attenuation of surface waves due to turbulence has been investigated, for example, by Skoda (1972), Green et al. (1972), van Hoften \& Karaki (1976) and Kitaigorodskii \& Lumley (1983). In their theoretical study, Kitaigorodskii \& Lumley identified a wave decay mechanism involving the transport of wave energy away from the surface by the turbulent velocity field, and parameterised that process in terms of the friction velocity of the turbulence. However, they emphasised that this process is only significant in a random wave field, and not for a periodic wave, because it requires a non-zero correlation between the turbulent and the wave motions. Note how, in (3.22), what is necessary for the wave energy to decrease is a correlation between the wave velocity and the turbulent shear stress. The existence of this correlation is provided by the modulation of the shear stress over the wave cycle. Hence the present mechanism complements the mechanism proposed by Kitaigorodskii \& Lumley.

Skoda (1972), Green et al. (1972) and van Hoften \& Karaki (1976) performed experiments where they measured the decay of approximately monochromatic, mechanically generated waves, due to turbulence induced by rotating paddles, an oscillating grid and channel bottom friction, respectively. Ölmez and Milgram (1992) studied the decay of periodic waves due to grid-generated turbulence, and re-analysed the data of Skoda (1972). They present an extensive list of the parameters of these experiments, including the intensity and length scale of the turbulence. They suggest an empirical formula for the temporal attenuation rate, which in the present notation is

$$
\beta_{t}^{O M}=0.103 \frac{u}{l^{\frac{1}{3}} \lambda_{w}^{\frac{2}{3}}}
$$

They adjusted the constant 0.103 to fit their experimental data. We note that the integral length scale of the turbulence, $l$, which appears in (3.25) does not appear in the RDT scaling (3.24) 


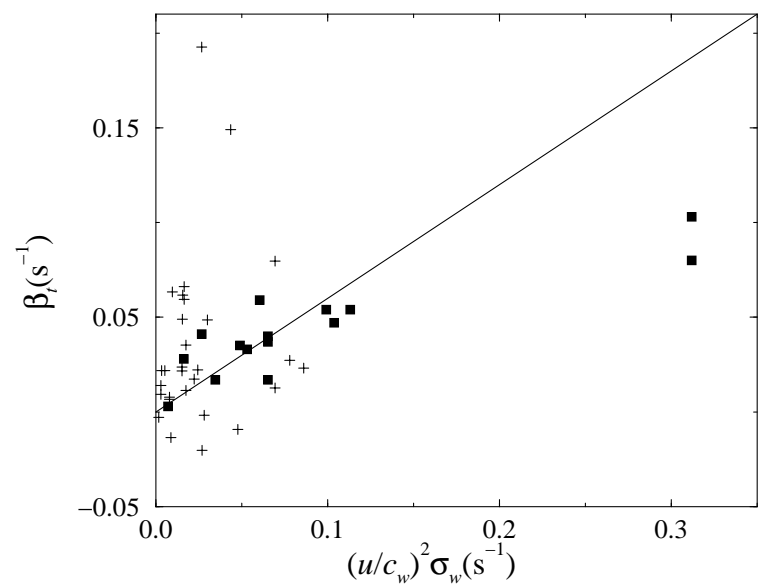

Figure 17. Comparison with experimental data of the temporal wave attenuation rate predicted by theory. Solid line: equation (3.24) with $a=0.6$, squares: data from Ölmez \& Milgram (1992), crosses: data from Skoda (1972) (taken from Ölmez \& Milgram 1992).

because, according to the RDT model developed here, the integral length scale does not affect the distortion by the Stokes drift, only the blocking of the turbulence by the boundary.

Figure 17 shows experimental data from Ölmez \& Milgram (1992) and Skoda (1972) for the temporal attenuation rate of surface waves plotted as a function of $\left(u / c_{w}\right)^{2} \sigma_{w}$. The straight line corresponds to the formula (3.24) with a best fit value of $a=0.6$. Figure 17 contains 2 data points from Skoda, corresponding to the highest decay rates, that do not appear in figure 11 of Ölmez \& Milgram (1992). We know of no reason to reject these points so they are included here, although they add considerably to the scatter. The large scatter in the data is not surprising since measured values of the attenuation rate due to turbulence are calculated as a residue of the wave decay due to other processes (e.g. geometric spreading in Ölmez \& Milgram's axisymmetric experiments, or channel wall friction in Skoda's experiments), and is thus subject to a large measurement error.

Another possible cause for the scatter is that the data of Ölmez \& Milgram and of Skoda do not satisfy strictly the assumptions of RDT on which the present scaling is based. These assumptions are that the wavelength is larger than the length scale of the turbulence and that the strain associated with the wave is larger than the strain by the turbulence itself, as expressed mathematically in (2.3). Except for one extreme measurement (which has $a_{w} k_{w} \sigma_{w} l / u=0.399$ ), the data measured by Ölmez \& Milgram (1992) have

$$
4.36<\frac{\lambda_{w}}{l}<7.10, \quad \text { and } \quad 0.583<\frac{a_{w} k_{w} \sigma_{w}}{u / l}<2.663 .
$$


Hence the turbulence in the experiments is small scale, but the straining by the wave is not always much larger than the straining of the turbulence by itself. The data of Skoda (1972) yield

$$
1.57<\frac{\lambda_{w}}{l}<10.296
$$

which probably satisfies the condition that the turbulence be small scale. We were unable to obtain values of wave slope for Skoda's data.

Notwithstanding these uncertainties, the great majority of the data points (40 in total) roughly align with the theoretical line. The scatter here is large, but is certainly no worse than in figure 11 of Ölmez \& Milgram (1992). This gives some support for the theoretical RDT scaling developed here and its variation with both properties of the turbulence and the surface wave, which both vary in the data shown in figure 17.

We now further compare the theoretical scaling (3.24) with the data of Green et al. (1972), who passed surface waves of varying wavelengths over grid-generated turbulence and measured the spatial attenuation of the wave amplitude. This comparison focuses then on the variation of wave damping rate with the surface wave properties.

To compare the RDT scaling with this data, the spatial attenuation rate is obtained from the temporal attenuation rate using the group velocity, $c_{g}=\mathrm{d} \sigma_{w} / \mathrm{d} k_{w}$, to give

$$
\beta_{x}=\beta_{t} / c_{g}
$$

Using $(3.28),(3.24)$ and the dispersion relation of free surface gravity waves, $\sigma_{w}^{2}=g k_{w}$, the spatial attenuation rate takes the form

$$
\beta_{x}=2 a \frac{u^{2}}{g^{3}} \sigma_{w}^{4}=2(2 \pi)^{4} a \frac{u^{2}}{g^{3}} f_{w}^{4},
$$

where $f_{w}=1 / T=\sigma_{w} / 2 \pi$ is the frequency of the waves (in $\mathrm{Hz}$ ). Hence, according to (3.29), the spatial decay rate is proportional to the fourth power of the frequency of gravity waves. We note that the empirical correlation suggested by Ölmez \& Milgram, given in (3.25), yields $\beta_{x} \propto f_{w}^{7 / 3}$, a very different dependence on wave frequency.

We estimate that for the Green et al. experiments the turbulent RMS velocity took the value $u=1.2 \mathrm{~cm} \mathrm{~s}^{-1}$. This value was chosen because it is mentioned at the beginning of Green et al.'s paper that the grid that generates the turbulence oscillates at a frequency of $\approx 1 \mathrm{~Hz}$ and produces 
eddies of $\approx 1 \mathrm{~cm}$ size. Figure 3 of Green et al. (1972) would suggest a much higher value of $u$, but that figure is probably in error, since Green et al. refer 'serious problems associated with variations of the probe sensitivity with flow direction'. A value $u=O\left(1 \mathrm{~cm} \mathrm{~s}^{-1}\right)$ is corroborated by other experiments using similar configurations of oscillating grids (e.g. Brumley \& Jirka 1987; Ölmez \& Milgram 1992; Kit et al. 1997).

Finally, the numerical values of the damping rates plotted in figure 4 of Green et al. (1972) are two orders of magnitude higher than the more recent results of Milgram (1998). This observation suggests that the axis label in figure 4 of Green et al. is in error and should be measured in $\mathrm{m}^{-1}$ instead of the $\mathrm{cm}^{-1}$ written on the figure. This mistake has been corrected in replotting their data in figure 18.

Figure 18 compares the RDT scaling (3.29) and the data of Green et al. (1972). Green et al. considered the decay of three wave types. Firstly, they performed experiments with no wind and measured the attenuation of paddle-generated waves, consisting of a fundamental mode and bound harmonics. Secondly, they measured the spatial attenuation of wind-generated waves. The data from the fundamental modes of paddle-generated waves, which are denoted by filled circles in figure 18 , are closest to the conditions of the model developed here. Indeed the agreement between the RDT scaling with the same value of $a=0.6$ and these data is encouraging. We note that the empirical correlation of Ölmez and Milgram (1992), (3.25), yields a spatial attenuation that varies as $f_{w}^{7 / 3}$, which does not agree well with the data in figure 18. The data obtained by Green et al. for the bound harmonics and the wind-generated waves are also shown for completeness as crosses and triangles, despite the obvious complications associated with these data. These data too agree in order of magnitude with the RDT scaling. We conclude that the RDT scaling shows a variation with wave properties that is consistent with available data.

How does the magnitude of the present wave decay mechanism compare with the rate of growth due to wind forcing, which has roughly the same form? These mechanisms have been studied extensively by Miles (1957), van Duin \& Janssen (1992) and Belcher \& Hunt (1993), among others, and can be encapsulated in the formula of Plant (1982), which when written in terms of the growth 
On the distortion of turbulence by a progressive surface wave

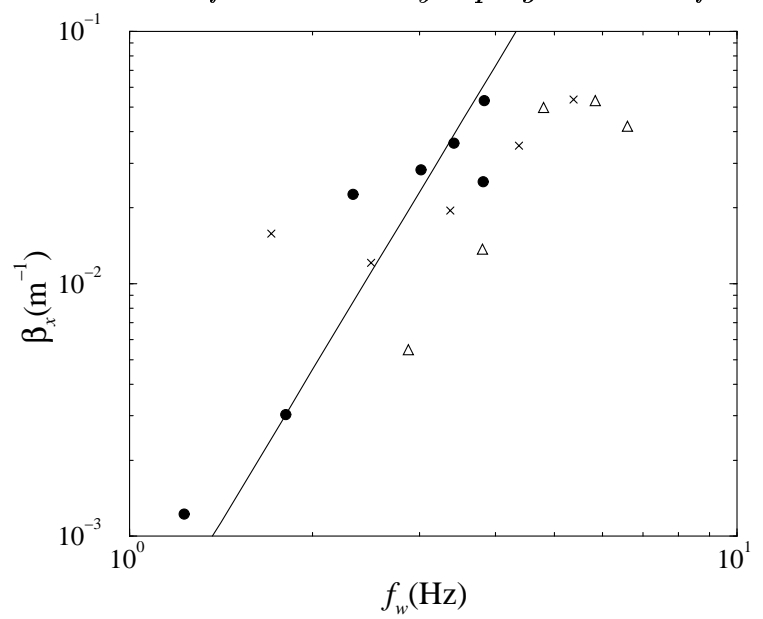

Figure 18. Comparison with experimental data of the spatial wave attenuation rate predicted by theory. Solid line: equation (3.29) with $a=0.6$ and $u=1.2 \mathrm{~cm} \mathrm{~s}^{-1}$, circles: paddle-generated fundamentals, crosses: second harmonics, triangles: wind generated waves.

rate of wave amplitude gives

$$
\gamma=16 \frac{\rho_{a}}{\rho_{w}}\left(\frac{u_{* a}}{c_{w}}\right)^{2} \sigma_{w},
$$

where $\rho_{a}$ is the density of air, $\rho_{w}$ is the density of water and $u_{* a}$ is the friction velocity in the air. Often it is the growth of wave energy that is quoted, which has a growth rate twice that given in (3.30). It should be noted that, since it was obtained from the rate of change of wave amplitude with time, (3.30) not only reflects the positive input of energy into the waves due to the wind, but also any dissipative processes present.

In order to compare (3.24) with $(3.30)$, it is necessary to relate the turbulence intensity in the water, $u$, to the friction velocity in the air. Experiments by Magnaudet \& Masbernat (1990) and Thais \& Magnaudet (1996) suggest that the turbulent RMS velocity in water containing turbulence and surface waves is $u \approx 3 u_{* w}$ near the surface. On the other hand, Thais \& Magnaudet (1996) and Belcher et al. (1994) have noted that the friction velocity in the water is related to the friction velocity in the air by $\rho_{a} u_{* a}^{2}=\rho_{w} u_{* w}^{2}$ due to continuity of the turbulent stress at the interface. With these two relations, and using also $a=0.6,(3.24)$ yields

$$
\beta_{t} \approx 5.4 \frac{\rho_{a}}{\rho_{w}}\left(\frac{u_{* a}}{c_{w}}\right)^{2} \sigma_{w},
$$

showing, by comparison with (3.30), that the decay rate due to turbulence in the water is approximately a factor of 3 smaller than the amplitude growth rate due to forcing by the wind. In their 
model of coupled turbulent air-water flow, Belcher et al. (1994) evaluated the effect of turbulence in the water on the growth of surface waves, concluding that the growth rate could be reduced by a factor of about 2 . They considered that only the part of the flow within an 'inner region', where the turbulence is in a local equilibrium (very near the boundary). The present calculation shows that the 'outer' part of the flow, where the turbulence is rapidly distorted, contributes to a non-negligible reduction of the wave growth rate.

Finally, the wave decay mechanism estimated in this subsection is only important at wavenumbers for which viscous dissipation does not dominate. Bearing in mind that the wave attenuation rate due to viscous dissipation is $2 \nu k_{w}^{2}$ (Lamb 1932), where $\nu$ is the kinematic viscosity of the water, the present mechanism is relevant when

$$
a \frac{u^{2}}{c_{w}^{2}} \sigma_{w} \gg 2 \nu k_{w}^{2} \quad \Rightarrow \quad u \gg\left(2 \nu \sigma_{w} / a\right)^{\frac{1}{2}} .
$$

Taking $\sigma_{w}=1 \mathrm{~s}^{-1}$ and noting that $\nu=1 \times 10^{-6} \mathrm{~m}^{2} \mathrm{~s}^{-1}$ and $a=0.6$, it is concluded that $u$ must be considerably larger than $2 \mathrm{~mm} \mathrm{~s}^{-1}$ (not a difficult condition to satisfy in the ocean). Alternatively, if $u=2 \mathrm{~cm} \mathrm{~s}^{-1}$ is assumed, gives $\sigma_{w} \ll 100 \mathrm{~s}^{-1}$, corresponding approximately to $\lambda_{w} \gg 1.5 \mathrm{~cm}$. Wavelengths outside this range would be excluded anyway because of the condition requiring the scale of the wave to be much larger than the scale of the turbulence (first equation of (2.3)). The mechanism addressed here is therefore primarily a wave attenuation mechanism for gravity waves.

\section{Conclusions}

Previous scaling arguments, developed by Belcher et al. (1994), have shown that turbulence below a thin 'inner region' in the ocean surface layer is subjected to rapid distortion by a surface wave. Hence in this paper we have developed a rapid-distortion model to investigate the interactions between initially homogeneous, shear-free turbulence and a progressive, irrotational surface wave. The model is applicable when the integral length scale of the turbulence is much smaller than the wavelength of the wave and the slope of the wave is sufficiently high that the straining of the turbulence by the wave is stronger than the straining of the turbulence by itself.

The periodic orbital motion of the wave modulates the turbulence over a wave cycle such that the streamwise Reynolds stress attains maxima at the wave crests and minima at the wave troughs, 
and the normal Reynolds stress attains maxima at the wave troughs and minima at the crests. This behaviour is consistent with the experimental results of Thais \& Magnaudet (1996).

Over several wave cycles the turbulence is made strongly anisotropic as the Stokes drift associated with the wave tilts the vertical component of the turbulent vorticity into the horizontal, and subsequently amplifies it as streamwise vorticity. As this process occurs the streamwise Reynolds stress decreases, while the spanwise and normal Reynolds stresses increase over time at roughly the same rate. The integral length scales of the turbulence indicate the structures that develop under the progressive action of the Stokes drift. The streamwise velocity fluctuations remain isotropic when viewed in horizontal planes parallel to the surface, whereas normal velocity fluctuations become elongated in the streamwise direction and so too do the spanwise velocity fluctuations, although to a lesser extent than the normal fluctuations. These properties are summarised in table 1. We interpret these results as the statistical signature of elongated streamwise vortices in the flow.

These results for turbulence distorted by Stokes drift are strikingly different to the corresponding results for turbulence distorted by a mean shear current (see table 1). When turbulence is distorted by a mean shear the streamwise Reynolds stress becomes the largest of the stresses and the structure of the streamwise velocity fluctuations becomes elongated in the streamwise direction. These are the statistical signatures of 'streaky structures' which have been identified in, for example, the DNS of Lee et al. (1990). We attribute the striking differences between the two sets of results to the absence of mean vorticity in the distortion by Stokes drift. The mean shear flow has spanwise mean vorticity which is distorted by turbulent velocity fluctuations, which then largely cancels the streamwise vorticity generated by stretching turbulent vorticity by the mean shear flow. These two processes thus cancel any tendency to produce streamwise vortices in the shear flow (see figure 15). When the turbulence is distorted by Stokes drift, however, there is no such cancellation and streamwise vortices emerge. This cancellation idea probably explains why, in their LES simulations, McWilliams et al. (1997) observe a continuous progression from shear turbulence to fully developed Langmuir turbulence: as the Stokes drift is increased and the mean shear is less and less able to cancel the tendency to produce streawise vortices. 


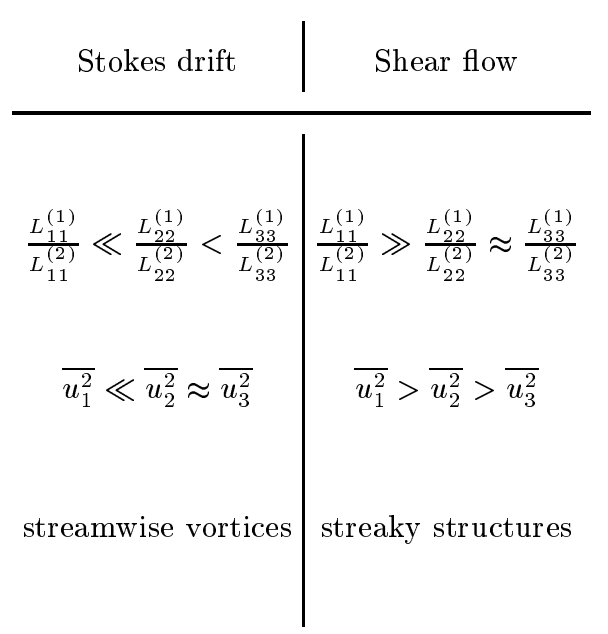

TABLE 1. Measures of the anisotropy of the flow structure in turbulence distorted by the Stokes drift of a wave and turbulence distorted by a mean shear flow.

It is interesting to compare these results from linear rapid-distortion theory with results obtained from fully nonlinear computations, such as the LES of 'Langmuir turbulence' performed by McWilliams et al. (1997). It was perhaps intuitively clear that homogeneous turbulence distorted by Stokes drift yields streamwise vortices (as discussed in the introduction to McWilliams et al. 1997). The detailed calculations confirm this expectation, but also give quantitative information. In particular, the RDT model produces the ordering of the components of the Reynolds stress and integral length scales, which are summarised in table 1. It is noteworthy that the ordering of both the components of the Reynolds stress and the indicators of anisotropy in the velocity fluctuations all agree with the orderings inferred from the LES calculations of McWilliams et al. (1997). This suggests that in Langmuir turbulence, as simulated by McWilliams et al., the wave-turbulence interaction dominates over the shear effects.

The generation of streamwise vortices by distortion of the turbulence by the Stokes drift also generates a negative shear stress in the turbulence. This shear stress does work against the fluctuating strain associated with the wave orbital motions leading to growth of the TKE. The time scale for this growth, found from scaling the TKE budget, is of $O\left(1 /\left(a_{w}^{2} k_{w}^{2} \sigma_{w}\right)\right)$. The energy going into the TKE is lost at precisely the same rate by the wave, which therefore decays in time. The 
amplitude of the waves decays exponentially with a temporal attenuation rate

$$
\beta_{t}=0.6\left(\frac{u}{c_{w}}\right)^{2} \sigma_{w} .
$$

The decay of wave energy would be twice this value for the amplitude decay. This decay rate was found to be consistent with the laboratory data of Ölmez \& Milgram (1992) and Green et al. (1972). Hence we have established a definite connection between decay of waves and growth of turbulence.

This work raises the possibility that subsurface turbulence in the ocean, generated by breaking waves or shear instability, provides the vertical vorticity from which Stokes drift generates streamwise vortices, i.e. Langmuir circulations. This possibility complements the CL2 instability mechanism (Craik \& Leibovich 1976), where the initial vertical vorticity is provided by spanwise variations in the mean shear current. LES, which can resolve all three components of the flow, namely the mean shear flow, turbulence and Stokes drift, offers probably the best vehicle for establishing the relative contributions of these two processes.

M. A. C. T. acknowledges the financial support of the portuguese Fundação para a Ciência e Tecnologia (FCT), under grant PraxisXXI/BD/9401/96.

\section{REFERENCES}

Agrawal, Y. C., Terray, E. A., Donelan, M. A., Hwang, P. A., Williams, A. J., Drennan, W. M., Kahma, K. K. and Kitaigorodskit, S. A. 1992 Enhanced dissipation of kinetic-energy beneath surface-waves. Nature, 359, 219-220.

Batchelor, G. K. and Proudman, I. 1954 The effect of rapid distortion of a fluid in turbulent motion. Quart. J. Mech. Appl. Math., 7, 83-103.

Belcher, S. E. And Hunt, J. C. R. 1993 Turbulent shear flow over slowly moving waves. J. Fluid Mech., 251, 109-148.

Belcher, S. E. And Hunt, J. C. R. 1998 Turbulent flow over hills and waves. Ann. Rev. Fluid Mech., 30, 507-538.

Belcher, S. E., Harris, J. A. And Street, R. L. 1994 Linear dynamics of wind waves in coupled turbulent air flow. Part 1. Theory. J. Fluid Mech., 271, 119-151.

Britter, R. E., Hunt, J. C. R. And Richards, K. J. 1981 Air flow over a two-dimensional hill: studies of velocity speed-up, roughness effects and turbulence. Q. J. Roy. Met. Soc., 107, 91-110. 
Brocchini, M. and Peregrine, D. H. 2000 The dynamics of turbulence at free surfaces. Part 1. Description of strong turbulence at a free surface. J. Fluid Mech. (submitted).

Brumley, B. H. AND JiRKA, G. 1987 Near-surface turbulence in a grid-stirred tank. J. Fluid Mech., 183, 235-263.

Craik, D. D. And Leibovich, S. 1976 A rational model for Langmuir circulations. J. Fluid Mech., 73, 401-426.

Duin, C. A. van And Janssen, P. A. E. M. 1992 An analytical model of the generation of surface gravity waves by turbulent air flow. J. Fluid Mech., 236, 197-215.

Durbin, P. A. 1978 Rapid distortion theory of turbulent flows. PhD thesis, University of Cambridge.

Durbin, P. A. 1981 Distorted turbulence in axisymmetric flow. Q. J. Mech. Appl. Math., 34, 489-500.

Durbin, P. A. And Hunt, J. C. R. 1980 On surface pressure fluctuations beneath turbulent flow round bluff bodies. J. Fluid Mech., 100, 161-184.

Eames, I. And MCIntyre, M. E. 1999 On the connection between Stokes drift and Darwin drift. Math. Proc. Camb. Phil. Soc., 126, 171-174.

Faller, A. J. AND Auer, S. J. 1988 The roles of Langmuir circulations in the dispersion of surface tracers. J. Phys. Oceanogr., 18, 1108-1123.

Gartshore, I. S., Durbin, P. A. And Hunt, J. C. R. 1983 The production of turbulent stress in a shear flow by irrotational fluctuations. J. Fluid Mech., 137, 307-329.

Green, T., Medwin, H. And Paquin, J. E. 1972 Measurements of surface wave decay due to underwater turbulence. Nature Phys. Sci., 237, 115-117.

Hoften, J. D. A. VAn And KARAKi, S. 1976 The interaction of gravity waves and turbulent channel flow. Technical Report, Department of Civil Engineering, Colorado State University.

Hunt, J. C. R. 1973 A theory of flow round two-dimensional bluff bodies. J. Fluid Mech., 61, 625-706.

Hunt, J. C. R. And Graham, J. M. R. 1978 Free-stream turbulence near plane boundaries. J. Fluid Mech., 84, 209-235.

Kit, E. L. G., Strang, E. J. and Fernando, H. J. S. 1997 Measurement of turbulence near shear-free density interfaces. J. Fluid Mech., 334, 293-314.

Kitaigorodskit, S. A. And Lumley, J. L. 1983 Wave-turbulence interactions in the upper ocean. Part I: The energy balance on the interacting fields of surface wind waves and wind-induced three-dimensional turbulence. J. Phys. Oceanogr., 13, 1977-1987.

Kitaigorodskit, S. A., Donelan, M. A., Lumley, J. L. And Terray, E. A. 1983 Wave-turbulence interactions in the upper ocean. Part II: Statistical characteristics of wave and turbulent components of the random velocity field in the marine surface layer. J. Phys. Oceanogr., 13, 1988-1999. 
Kline, S. J., Reynolds, W. C., Schtrauss, F. A. And Runstadler, P. W. 1967 The structure of turbulent boundary layers. J. Fluid Mech., 30, 741-773.

LAmB, H. 1932 Hydrodynamics. 6th edition. Cambridge University Press.

Lee, M. J. And Hunt, J. C. R. 1989 The structure of sheared turbulence near a plane boundary. 7th Simp. on Turbulent Shear Flows, Stanford.

LeE, M. J., Kim, J. And Moin, P. 1990 Structure of turbulence at high shear rate. J. Fluid Mech., 216, $561-583$

Leibovich, S. 1983 The form and dynamics of Langmuir circulations. Ann. Rev. Fluid Mech., 15, 391-427.

Longuet-Higgins, M. S. 1984 New integral relations for gravity waves of finite amplitude. J. Fluid Mech., 149, 205-215.

Longuet-Higgins, M. S. 1996 Surface manifestations of turbulent flow. J. Fluid Mech., 308, 15-29.

Magnaudet, J. And Masbernat, L. 1990 Interaction des vagues de vent avec le courant moyen et la turbulence. C. R. Acad. Sci. Paris, 311, 1461-1466.

MAnN, J. 1994 The spatial structure of neutral atmospheric surface-layer turbulence. J. Fluid Mech., 273, $141-168$

McLeish, W. And Putland, G. E. 1975 Measurements of wind-driven flow profiles in the top millimeter of water. J. Phys. Oceanogr., 5, 516-518.

MCWilliams, J. C., Sullivan, P. P. And Moeng, C.-H. 1997 Langmuir turbulence in the ocean. J. Fluid Mech., 334, 1-30.

Melville, W. K. 1996 The role of surface-wave breaking in air-sea interaction. Ann. Rev. Fluid Mech., 28, 279-321.

Melville, W. K., Shear, R. And Veron, F. 1998 Laboratory measurements of the generation and evolution of Langmuir circulations. J. Fluid Mech., 364, 31-58.

Miles, J. W. 1957 On the generation of surface waves by shear flow. J. Fluid Mech., 3, 185-204.

Milgram, J. H. 1998 Short wave damping in the simultaneous presence of a surface film and turbulence. J. Geophys. Res., 103, 15717-15727.

Nazarenko, S., Kevlahan, N. K.-R. and Dubrulle, B. 1999 WKB theory for rapid distortion of inhomogenous turbulence. J. Fluid Mech., 390, 325-348.

Nepf, H. M., Cowen, E. A., Kimmel, S. J. And Monismith, S. G. 1995 Longitudinal vortices beneath breaking waves. J. Geophys. Res., 100, 16211-16221.

Ölmez, H. S. AND Milgram, J. H. 1992 An experimental study of attenuation of short water waves by turbulence. J. Fluid Mech., 239, 133-156.

Phillips, O. M. 1957 On the generation of waves by turbulent wind. J. Fluid Mech., 2, 417-445. 
Phillips, O. M. 1959 The scattering of gravity waves by turbulence. J. Fluid Mech., 5, 177-192.

Phillips, O. M. 1977 The dynamics of the upper ocean. Cambridge University Press.

Plant, W. J. 1982 A relationship between wind shear stress and wave slope. J. Geophys. Res., 87, 1961-1967.

Rapp, R. And Melville, W. 1990 Laboratory measurements of deep-water breaking waves. Philos. Trans. Roy. Soc. London A, 331, 735-800.

Skoda, J. D. 1972 The interaction of waves and turbulence in water. Ph.D. thesis. University of California, Berkeley.

TeiXeira, M. A. C. 2000 Interaction of turbulence with a free surface. Ph.D. thesis. University of Reading.

Terray, E. A., Donelan, M. A., Agrawal, Y. C., Drennan, W. M., Kahma, K. K., Williams, A. J., Hwang, P. A. And Kitaigorodskit, S. A. 1996 Estimates of kinetic energy dissipation under breaking waves. J. Phys. Oceanogr., 26, 792-807.

Thais, L. And Magnaudet, J. 1996 Turbulent structure beneath surface gravity waves sheared by the wind. J. Fluid Mech., 328, 313-344.

Townsend, A. A. 1970 Entrainment and the structure of turbulent flow. J. Fluid Mech., 41, 13-46.

Townsend, A. A. 1976 The structure of turbulent shear flow, 2nd edition. Cambridge University Press. 